TRANSACTIONS OF THE

AMERICAN MATHEMATICAL SOCIETY

Volume 359, Number 8, August 2007, Pages 3933-3972

S 0002-9947(07)04136-0

Article electronically published on February 23, 2007

\title{
UNIFORMIZABLE FAMILIES OF $t$-MOTIVES
}

\author{
GEBHARD BÖCKLE AND URS HARTL
}

\begin{abstract}
Abelian $t$-modules and the dual notion of $t$-motives were introduced by Anderson as a generalization of Drinfeld modules. For such Anderson defined and studied the important concept of uniformizability. It is an interesting question and the main objective of the present article to see how uniformizability behaves in families. Since uniformizability is an analytic notion, we have to work with families over a rigid analytic base. We provide many basic results, and in fact a large part of this article concentrates on laying foundations for studying the above question. Building on these, we obtain a generalization of a uniformizability criterion of Anderson and, among other things, we establish that the locus of uniformizability is Berkovich open.
\end{abstract}

\section{INTRODUCTION}

Over the complex numbers $\mathbb{C}$, any elliptic curve $E$ is biholomorphically isomorphic to a quotient $\mathbb{C} / \Lambda$, where $\Lambda$ is the period lattice of $E$. The analogue of the complex numbers in characteristic $p$ is the field $\mathbb{C}_{\infty}$ which is the topological closure of an algebraic closure of $\mathbb{F}_{q}((1 / t))$. With this analogy in mind, Drinfeld associated to any discrete finitely generated $\mathbb{F}_{q}[t]$-sublattice $\Lambda$ of $\mathbb{C}_{\infty}$ an algebraic object, which we now call a Drinfeld-module.

In the case of elliptic curves, the uniformization $\mathbb{C} / \Lambda$ is not just a point-wise property. For any complex analytic family $E \rightarrow S$ of elliptic curves over a complex manifold $S$, if $E_{0}$ over a point $s_{0}$ is given by a lattice $\Lambda_{0}$, then this construction may be extended locally analytically to a lattice over an open neighborhood of $s_{0}$ in $S$. In fact, there is a tautological analytical local system $\mathbb{L}:=\left(\Lambda_{z} \subset \mathbb{C}\right)_{z \in \mathbb{H}}$ on the upper half plane $\mathbb{H}$ which represents a uniformization of the universal family of elliptic curves on $\mathbb{H}$. Now for any simply connected neighborhood $U \subset S$ of $s_{0}$, there exists an analytic morphism $U \rightarrow \mathbb{H}$ such that $E$ restricted to $U$ is obtained from the universal family on $\mathbb{H}$ via pullback, and thus the corresponding local system is the pullback of $\mathbb{L}$. The same can be done for Drinfeld-modules of rank $r$ using the rigid analytic space $\Omega^{r}$ instead of $\mathbb{H}$, and where one considers the rigid analytic Grothendieck topology instead of the complex analytic one; cf. [9] and [6], Chap. 4.

In [1], Anderson introduced the notion of a $t$-module, generalizing that of a Drinfeld-module. Again there arises the question of the uniformizability of these

Received by the editors November 15, 2004 and, in revised form, July 21, 2005.

2000 Mathematics Subject Classification. Primary 11G09; Secondary 14G22.

Key words and phrases. Drinfeld modules, higher dimensional motives, rigid analytic geometry.

(C)2007 American Mathematical Society Reverts to public domain 28 years from publication 
objects, i.e., the question of whether one can always write these in a suitable sense as $\mathbb{C}_{\infty}^{d} / \Lambda$ for some discrete lattice $\Lambda$. As Anderson shows, not all $t$-modules can be written in such a way. If they can be, he calls them uniformizable, and moreover any uniformizable $t$-module has good reduction.

When compared with the classical situation, the nature of uniformizability cannot be explained satisfactorily. One thinks of $t$-modules as being analogous to abelian varieties. But over $\mathbb{C}$ any abelian variety is isomorphic to $\mathbb{C}^{g}$ modulo a suitable discrete lattice of full rank $2 g$. Over a $p$-adic complete algebraically closed field $\mathbb{C}_{p}$ only abelian varieties with bad, but totally multiplicative reduction admit a uniformization by a discrete lattice.

To any $t$-module, Anderson associates a dual object called $t$-motive, and the uniformizability of the $t$-module is equivalent to the analytic triviality of the associated $t$-motive. We explain the correspondence in the last section of the article. Moreover $t$-motives are a special case of so-called $\tau$-sheaves. The aim of this article is to study analytic triviality in families of $\tau$-sheaves.

The first example of a family of $\tau$-sheaves arises from a family of Drinfeld modules on a $\mathbb{C}_{\infty}$-variety $X$. Since all Drinfeld modules are uniformizable, every fiber of this family is analytically trivial. Moreover, as we have remarked above, the family of Drinfeld modules can even be uniformized as a whole on a covering of $X$. However, this covering will in general not be a variety but a rigid analytic space.

Beyond Drinfeld modules, the next interesting examples were constructed by Gardeyn in his thesis; cf. [12, II.2]. In Section 7 of this article, we describe another non-trivial family which was computed by R. Pink and which in fact describes a fine moduli space of pure "polarized" $t$-motives of rank and dimension 2 . In these families not every fiber is analytically trivial.

Given a family of $\tau$-sheaves on a variety $X$ over $\mathbb{C}_{\infty}$, the ultimate goal is to describe the nature of the locus of analytic triviality inside $X$. We expect that the equations (better: inequalities) describing analytic triviality are rarely algebraic and in the example of Section 7 this is indeed not the case. Therefore to investigate analytic triviality we are again urged to work in a rigid analytic setting. In this article we show that the locus of analytic triviality is Berkovich open; cf. Theorem 5.5. However the known examples suggest that in fact the locus of analytic triviality of a family of $\tau$-sheaves on an affinoid space is the complement of a quasi-compact rigid analytic subspace. This is a much stronger assertion.

To fix ideas, let $R$ denote an affinoid algebra over $\mathbb{C}_{\infty}$, and let $(M, \tau)$ be a pair of a finitely generated projective module $M$ over the Tate-algebra $R\langle t\rangle$ over $R$, and an endomorphism $\tau: M \rightarrow M$ such that $\tau\left(r t^{i} m\right)=t^{i} r^{q} \tau(m)$ for $r \in R$ and $m \in M$. (This is the affine version of a rigid analytic $\tau$-sheaf; cf. Definition 1.2.) The module $M$ gives rise to a quasi-coherent sheaf $\mathcal{F}$ on $X:=\operatorname{Sp} R$, and $\tau$ is an $\mathbb{F}_{q}[t]$-linear operation on it. Let $W(\mathcal{F})$ denote the associated étale sheaf on the étale site over $X$, and consider the left exact fundamental sequence

$$
0 \longrightarrow \underline{\mathcal{F}}^{\tau} \longrightarrow W(\mathcal{F}) \stackrel{1-\tau}{\longrightarrow} W(\mathcal{F}),
$$

which defines the étale sheaf $\underline{\mathcal{F}^{\tau}}$. The latter is a sheaf of $\mathbb{F}_{q}[t]$-modules. Its construction is reminiscent of Artin-Schreier theory (and specializes to it, for instance for $t \mapsto 0)$. The pair $(M, \tau)$ is called analytically trivial if étale locally the rank of $\underline{\mathcal{F}}^{\tau}$ as a free module over $\mathbb{F}_{q}[t]$ is the same as the rank of $M$ over $R\langle t\rangle$. 
It turns out that $\underline{\mathcal{F}}^{\tau}$ is overconvergent in the sense of [18. Therefore, we work on the rigid analytic site not just with the usual classical points, but with so-called analytic points, first defined by van der Put and later developed by Berkovich. These points can detect exactness in sequences of overconvergent sheaves. Some necessary definitions on this are recalled in the Appendix. In Section 1 we define our basic objects of study - rigid analytic $\tau$-sheaves. Equipped with these tools and definitions, we shall prove the following results:

In Section 3 we establish a key lemma on the exact sequence (0.1). Its first consequence is the overconvergence of $\underline{\mathcal{F}}^{\tau}$. It also implies that the sequence is right exact if and only if it is so at all étale analytic stalks. Moreover the $\mathbb{F}_{q}[t]$-modules of sections of $\underline{\mathcal{F}}^{\tau}$ are free of rank at most that of $M$, and for $V \rightarrow U$ an étale cover with $V$ connected, the inclusion $\underline{\mathcal{F}}^{\tau}(U) \hookrightarrow \underline{\mathcal{F}}^{\tau}(V)$ is injective and saturated.

Then in Section 4, we investigate triviality of $\tau$-sheaves, where the pair $(M, \tau)$ is trivial, if $M$ is generated over $R\langle t\rangle$ by the global sections $\underline{\mathcal{F}}^{\tau}(X)$. For $R$ a field our results are translations of Anderson from [1]. The case of a point is the crucial step when we prove a simple condition for global triviality. Analytic triviality is clearly a weaker condition than triviality, and we study this concept in detail in the subsequent Section 5. There we prove that the analytically trivial locus is Berkovich open. We also show that on the one hand analytic triviality is equivalent to analytic triviality at all analytic stalks, and on the other that it is equivalent to the triviality of the $\tau$-sheaf over a suitable temperate (in the sense of [2]) étale covering.

In Section 6] we investigate natural conditions under which the sequence (0.1) is right exact. Essentially we require that the $\tau$-sheaf is point-wise composed of trivial and nilpotent $\tau$-sheaves. Section 7 is dedicated to an example by R. Pink in which he computes the analytically trivial locus of a 2-dimensional moduli space of $t$-modules which are not Drinfeld-modules.

The last section, Section 8 , describes the transition from $t$-modules to $\tau$-sheaves, describes the resulting dualities on the level of lattices, and formulates and proves Anderson's uniformizability criterion for families of $t$-motives. Here we also prove the existence of Anderson's exponential map over an arbitrary (rigid analytic) base.

\section{Rigid ANALYTiC $\tau$-SHEAVES}

In this section, we introduce our basic objects of study, namely rigid analytic families of $\tau$-sheaves. They were first introduced in [6] and form a natural generalization of analytic objects defined and studied first by Anderson in [1, § 2]. We recall those definitions and constructions which will be relevant to the question of analytic triviality, which is the central topic of this article.

1.1. Definitions. Let $\mathbb{F}_{q}$ be the field of $q$ elements and characteristic $p$, let $C$ be a complete, smooth, geometrically irreducible curve over $\mathbb{F}_{q}$, and $\infty$ a fixed closed point on $C$. Let $A$ be the ring of regular functions on $C \backslash\{\infty\}$ and $K$ its field of fractions. The completion of $K$ at the place $\infty$ is denoted $K_{\infty}$, and the completion of an algebraic closure of $K_{\infty}$ is denoted $\mathbb{C}_{\infty}$. For an $\mathbb{F}_{q}$-scheme $X$ denote by $\sigma_{X}$ its absolute Frobenius endomorphism with respect to $\mathbb{F}_{q}$ which acts as the identity on points and as the $q$-power map on the structure sheaf.

In the following definition, the $\operatorname{ring} \tilde{A}$ is either $A$ or $A / \mathfrak{a}$ for some non-zero ideal $\mathfrak{a}$ of $A$. 
Definition 1.1. For $X$ as above an (algebraic) $\tau$-sheaf over $\tilde{A}$ on $X$ is a pair $\underline{\mathcal{F}}:=\left(\mathcal{F}, \tau_{\mathcal{F}}\right)$ consisting of a coherent sheaf $\mathcal{F}$ on $X \times_{\mathbb{F}_{q}}$ Spec $\tilde{A}$ and an $\mathcal{O}_{X \times_{\mathbb{F}_{q}} \operatorname{Spec} \tilde{A}^{-}}$ module homomorphism

$$
\tau_{\mathcal{F}}:\left(\sigma_{X} \times \mathrm{id}\right)^{*} \mathcal{F} \longrightarrow \mathcal{F} .
$$

The notion of $\tau$-sheaf as above was first introduced in [7. An earlier version that required local freeness was defined in [27. In [7] it is also shown that the category of $\tau$-sheaves is stable under tensor product, and pullback and proper pushforward along morphisms in $X$.

As the present article centers around the notion of uniformizability, we need, as in 6], to pass from the above algebraic to a corresponding rigid analytic setting. (See Bosch, Güntzer, Remmert 8 for a general introduction to rigid analytic geometry.) Let $L$ be a complete non-archimedean valued field with $K_{\infty} \subset L \subset \mathbb{C}_{\infty}$. If $X$ is a scheme locally of finite type over $L$, we denote by $X^{\text {rig }}$ the associated rigid analytic space; cf. [8, $\S 9.3 .4]$. If $X$ is a scheme locally of finite type over $\mathbb{F}_{q}$, then we often simply write $X^{\text {rig }}$ for $\left(X \otimes_{\mathbb{F}_{q}} L\right)^{\text {rig. }}$. Corresponding to $X \mapsto X^{\text {rig }}$ one has a functor $\mathcal{F} \mapsto \mathcal{F}^{\text {rig }}$ on coherent sheaves. If one applies rigidification to an algebraic $\tau$-sheaf as above, it is a simple matter to verify that one will obtain what we are about to define, namely a rigid analytic $\tau$-sheaf. (In fact one obtains two, one for each of the coefficient rings we will introduce.)

Unless indicated otherwise, from now on by $X$ we denote a rigid analytic space on $L$ and by $\sigma_{X}$ the Frobenius on $X$ with respect to $\mathbb{F}_{q}$, i.e., $\sigma_{X}$ is the $q$-power map on affinoid algebras. We consider the following two natural coefficient rings:

(a) $A(\infty):=\Gamma\left(\mathfrak{A}(\infty), \mathcal{O}_{\mathfrak{A}(\infty)}\right)$ denotes the ring of entire functions on $\mathfrak{A}(\infty):=$ $(\operatorname{Spec} A)^{\mathrm{rig}}$.

(b) $A(1):=\Gamma\left(\mathfrak{A}(1), \mathcal{O}_{\mathfrak{A}(1)}\right)$ denotes the ring of rigid analytic functions on the "unit disc" $\mathfrak{A}(1)$ of $\mathfrak{A}(\infty)$, which is constructed as follows:

Let $\mathcal{O}_{L}$ be the valuation ring of $L$ and consider the formal completion of the scheme Spec $A \otimes_{\mathbb{F}_{q}} \mathcal{O}_{L}$ along its special fiber over the maximal ideal of $\mathcal{O}_{L}$. This is an admissible formal scheme over $\operatorname{Spf} \mathcal{O}_{L}$ in the sense of Bosch, Lütkebohmert [11. Then $\mathfrak{A}(1)$ is the rigid analytic $L$-space associated to this formal scheme.

Equivalently, one may also fix a monomorphism $\mathbb{F}_{q}[t] \rightarrow A$, denote by $L\langle t\rangle$ the Tate-algebra over $L$ in the variable $t$, and set $A(1):=A \otimes_{\mathbb{F}_{q}[t]} L\langle t\rangle$ and $\mathfrak{A}(1)=$ $\operatorname{Sp} A(1)$.

In the following $\mathfrak{C}$ will stand for either $\mathfrak{A}(\infty)$ or $\mathfrak{A}(1)$ and $\mathcal{A}$ for $\Gamma\left(\mathfrak{C}, \mathcal{O}_{\mathfrak{C}}\right)$. By $\sigma_{L, \mathfrak{C}}$ we denote the pullback of the Frobenius $\sigma_{\operatorname{Sp} L}$ on $L$ along $\mathfrak{C} \rightarrow \operatorname{Sp} L$, i.e., $\sigma_{L, \mathfrak{C}}$ acts as the $q$-power map on the coefficients in $L$ and as the identity on the variables of $A$. On $X \times_{L} \mathfrak{C}$ we define the endomorphism $\sigma_{X, \mathfrak{C}}:=\sigma_{X} \times \sigma_{L, \mathfrak{C}}$. It replaces $\sigma_{X} \times$ id in the rigid analytic setting.

Definition 1.2. Let $X$ be a rigid analytic space over $L$. A rigid analytic $\tau$-sheaf over $\mathcal{A}$ on $X$ is a pair $\underline{\mathcal{F}}=\left(\mathcal{F}, \tau_{\mathcal{F}}\right)$ consisting of a rigid analytic coherent sheaf $\mathcal{F}$

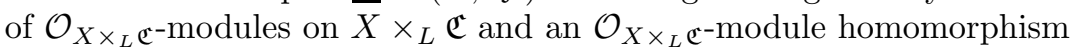

$$
\tau_{\mathcal{F}}: \sigma_{X, \mathfrak{C}}^{*} \mathcal{F} \longrightarrow \mathcal{F} \text {. }
$$

A homomorphism of rigid analytic $\tau$-sheaves over $\mathcal{A}$ on $X$ is a homomorphism of sheaves on $X \times{ }_{L} \mathfrak{C}$ which commutes with the action of $\tau$. It is a monomorphism, epimorphism, isomorphism, respectively, if its underlying homomorphism of sheaves on $X \times_{L} \mathfrak{C}$ has this property. 
A rigid analytic $\tau$-sheaf $\underline{\mathcal{F}}$ is called nilpotent, if there exists an $n \in \mathbb{N}$ such that $\tau^{n}:\left(\sigma_{X, \mathfrak{C}}^{n}\right)^{*} \mathcal{F} \longrightarrow \mathcal{F}$ is the zero homomorphism.

Examples 1.3. a) Let $\mathbb{1}_{X, \mathcal{A}}$ denote the rigid analytic $\tau$-sheaf over $\mathcal{A}$ on $X$ consisting of the structure sheaf $\mathcal{O}_{X \times_{L} \mathfrak{C}}$ and the natural isomorphism

$$
\sigma_{X, \mathfrak{C}}^{*} \mathcal{O}_{X \times_{L} \mathfrak{C}} \stackrel{\sim}{\longrightarrow} \mathcal{O}_{X \times_{L} \mathfrak{C}} .
$$

b) Let $\Omega^{r}:=\operatorname{Pr}^{r}\left(\mathbb{C}_{\infty}\right) \backslash\left\{K_{\infty}\right.$-rational hyper planes $\}$ be the $r$-dimensional Drinfeld upper half space and $E$ the universal Drinfeld-module on $\Omega^{r}$. To $E$ is associated an algebraic $\tau$-sheaf $(M(E), \tau)$ over $A$, namely the $A$-motive of $E$. The assignment is explained in Section 8 , and further details are given in [6], Chaps. 4 and 7.

A rigid analytic $\tau$-sheaf $\underline{\mathcal{F}}$ is called locally free if the underlying sheaf $\mathcal{F}$ is locally free on $X \times{ }_{L} \mathfrak{C}$, i.e., if there is an admissible covering $U_{i}, i \in I$, of $X \times_{L} \mathfrak{C}$ which trivializes $\mathcal{F}$. Both of the two examples above define in fact locally free $\tau$-sheaves. If further $X$ is connected, we denote the rank of $\mathcal{F}$ by rk $\mathcal{F}$.

One has the following important result of Lütkebohmert [21, Satz 1]:

Lemma 1.4. If $\mathcal{F}$ is a locally free sheaf on $X \times{ }_{L} \mathrm{Sp} L\langle t\rangle$, then there exists an admissible affinoid covering $U_{i}$ of $X$ such that $\left.\mathcal{F}\right|_{U_{i} \times \operatorname{Sp} L\langle t\rangle}$ is free on $U_{i} \times_{L} \operatorname{Sp} L\langle t\rangle$.

Using the above lemma, one may introduce local coordinates on locally free rigid analytic $\tau$-sheaves as follows:

Remark 1.5 (On local coordinates). Let $\underline{\mathcal{F}}$ be a locally free rigid analytic $\tau$-sheaf over $A(1)$ on $X$, and fix any non-constant homomorphism $\mathbb{F}_{q}[t] \rightarrow A$ (it will automatically be flat and finite). This induces a finite homomorphism $L\langle t\rangle \rightarrow A(1)$, making $A(1)$ into a free $L\langle t\rangle$-module. Thereby $\mathcal{F}$ becomes a locally free sheaf on $X \times_{L}$ Sp $L\langle t\rangle$, say of rank $r$. By Lemma 1.4, there exists an admissible affinoid covering of $X$ that trivializes $\mathcal{F}$, i.e., on each affinoid $\operatorname{Sp} B \subset X$ of this covering $\mathcal{F}$ is associated to a free module $M \cong B\langle t\rangle^{r}$. On the affinoid algebra $B$ we fix once and for all a complete $L$-algebra norm | |. For any matrix $\Delta$ with entries in $B$ we let $|\Delta|$ be the maximum over the norms of the entries. Choosing a basis $\left\{f_{1}, \ldots, f_{r}\right\}$ we write the elements of $M$ as vectors

$$
\sum_{n=0}^{\infty} b_{n} t^{n}, \quad b_{n} \in B^{r} \quad \text { with }\left|b_{n}\right| \rightarrow 0 \text { for } n \rightarrow \infty .
$$

We denote by $\sigma$ the action on $B\langle t\rangle$ given by $\sigma\left(\sum b_{n} t^{n}\right)=\sum\left(b_{n}\right)^{q} t^{n}$. Then the homomorphism $\tau_{\mathcal{F}}$ can be represented with respect to our basis by a matrix

$$
\tau_{\mathcal{F}}=\Delta \cdot \sigma, \quad \Delta=\sum_{n=0}^{\infty} \Delta_{n} t^{n} \in M_{r}(B\langle t\rangle)
$$

Hence $\Delta_{n} \in M_{r}(B)$ with $\left|\Delta_{n}\right| \rightarrow 0$ for $n \rightarrow \infty$. If we replace the basis vectors $f_{i}$ by $\widetilde{f}_{i}=\alpha f_{i}$ for $\alpha \in L^{\times}$, the $b_{n}$ are replaced by $\widetilde{b}_{n}=\alpha^{-1} b_{n}$ and therefore the matrices $\Delta_{n}$ are replaced by $\widetilde{\Delta}_{n}=\alpha^{1-q} \Delta_{n}$. Hence, we can adjust $\left|\Delta_{n}\right|$.

If there is an element $a \in A \backslash \mathbb{F}_{q}^{a l g}$ such that $\tau_{\mathcal{F}}$ is bijective on $\mathcal{F} / a \mathcal{F}$, and if we take $\mathbb{F}_{q}[t] \rightarrow A: t \mapsto a$ for the above homomorphism, then $\Delta_{0}$ will be in $\mathrm{GL}_{r}(B)$. Thus if we perform the finite étale base extension given by adjoining the solution $D$ of the equation $D=\Delta_{0}{ }^{\sigma} D$ and take the columns of $D$ as a new basis, we may further assume that $\Delta_{0}=\operatorname{Id}_{r}$. 
1.2. Functorial constructions. Change of coefficients $I$ :

Let $B$ be an affinoid $L$-algebra and consider $X=\operatorname{Spec} B$ as a scheme over $\mathbb{F}_{q}$. Let $\underline{\mathcal{F}}$ be an algebraic $\tau$-sheaf over $A$ on $X$. To it one naturally assigns a rigid analytic $\tau$-sheaf $\left(\mathcal{F}^{\text {rig }}, \tau_{\mathcal{F} \text { rig }}\right)$ over $A(\infty)$ on $\operatorname{Sp} B$ as follows: The underlying coherent rigid analytic sheaf $\mathcal{F}^{\text {rig }}$ is the pullback of $\mathcal{F}$ along the analytification morphism

$$
X \times_{L} \mathfrak{C}=\left(X \times_{\mathbb{F}_{q}} \operatorname{Spec} A\right)^{\mathrm{rig}} \longrightarrow X \times_{\mathbb{F}_{q}} \operatorname{Spec} A .
$$

On it $\tau_{\mathcal{F}}$ induces the homomorphism

$$
\tau_{\mathcal{F}^{\text {rig }}}: \sigma_{X, \mathfrak{C}}^{*} \mathcal{F}^{\text {rig }}=\left((\sigma \times \mathrm{id})^{*} \mathcal{F}\right)^{\text {rig }} \stackrel{\tau_{\mathcal{F}}^{\text {rig }}}{\longrightarrow} \mathcal{F}^{\text {rig }} .
$$

This construction generalizes in an obvious way to arbitrary rigid analytic spaces $X$.

Change of coefficients II:

Let $\underline{\mathcal{F}}$ be a rigid analytic $\tau$-sheaf over $A(\infty)$ on $X$. Then the action of $\tau_{\mathcal{F}}$ preserves the restriction $\left.\mathcal{F}\right|_{X \times_{L} \mathfrak{A}(1)}$, and so we obtain a $\tau$-sheaf over $A(1)$ on $X$, which we denote by $\underline{\mathcal{F}} \otimes_{A(\infty)} A(1)$.

Change of coefficients III:

Let $\underline{\mathcal{F}}$ be a rigid analytic $\tau$-sheaf over $A(1)$ on $X$. For any ideal $\mathfrak{a}$ of $A$ the ideal $\mathfrak{a} A(1) \subset A(1)$ is closed in the topology and satisfies $A / \mathfrak{a} \cong A(1) / \mathfrak{a} A(1)$. This identification induces an obvious functor from rigid analytic $\tau$-sheaves $\underline{\mathcal{F}}$ over $A(1)$ on $X$ to algebraic $\tau$-sheaves over $A / \mathfrak{a}$ on $X$, denoted by $\underline{\mathcal{F}} \mapsto \underline{\mathcal{F}} / \mathfrak{a} \underline{\mathcal{F}}$. If $\underline{\mathcal{F}}$ is a locally free rigid analytic $\tau$-sheaf over $A(1)$, then $\underline{\mathcal{F}} / \mathfrak{a} \mathcal{F}$ is a locally free algebraic $\tau$-sheaf over $A / \mathfrak{a}$.

Base change:

Let $\underline{\mathcal{F}}$ be a rigid analytic $\tau$-sheaf over $\mathcal{A}$ on $X$. Further let $\pi: Y \rightarrow X$ be a general morphism of rigid analytic spaces $Y$ over $L^{\prime}$ and $X$ over $L$ (cf. Definition A.9). Let

$$
\mathcal{A}^{\prime}:=\mathcal{A} \widehat{\otimes}_{L} L^{\prime}=\Gamma\left(\mathfrak{C}^{\prime}, \mathcal{O}_{\mathfrak{C}^{\prime}}\right), \quad \text { where } \mathfrak{C}^{\prime}:=\mathfrak{C} \widehat{\otimes}_{L} L^{\prime} .
$$

Then we define the rigid analytic $\tau$-sheaf $\pi^{*} \mathcal{F}$ over $\mathcal{A}^{\prime}$ on $Y$ to be the pulled back sheaf $\left(\pi \times \mathrm{id}_{\mathfrak{C}}\right)^{*} \mathcal{F}$ together with the composite homomorphism

$$
\sigma_{Y, \mathfrak{C}^{\prime}}^{*}\left(\pi \times \mathrm{id}_{\mathfrak{C}}\right)^{*} \mathcal{F}=\left(\pi \times \mathrm{id}_{\mathfrak{C}}\right)^{*} \sigma_{X, \mathfrak{C}}^{*} \mathcal{F} \stackrel{\left(\pi \times \mathrm{id}_{\mathfrak{C}}\right)^{*} \tau_{\mathcal{F}}}{\longrightarrow}\left(\pi \times \mathrm{id}_{\mathfrak{C}}\right)^{*} \mathcal{F} .
$$

In particular, every analytic point $x$ of $X$ (Definition A.1) gives rise to a general morphism $i: \operatorname{Sp} k(x) \rightarrow X$ (cf. Section A.1). The pullback $i^{*} \mathcal{F}$ is a $\tau$-sheaf on $\operatorname{Sp} k(x)$, which we also denote by $x^{*} \mathcal{F}$ in the following. For local sections $f$ of $\mathcal{F}$ we use the notation $f(x):=\left(i \times \operatorname{id}_{\mathfrak{C}}\right)^{*} f$ for the value of $f$ in $i^{*} \mathcal{F}$.

$\tau$-invariants $I$ :

A rigid analytic $\tau$-sheaf $\underline{\mathcal{F}}$ over $\mathcal{A}$ on $X$ comes with a homomorphism $\tau_{\mathcal{F}}$ : $\sigma_{X, \mathfrak{C}}^{*} \mathcal{F} \longrightarrow \mathcal{F}$. By adjunction between $\sigma_{X, \mathfrak{C}}^{*}$ and $\left(\sigma_{X, \mathfrak{C}}\right)_{*}$, we obtain a homomorphism

$$
\mathcal{F} \stackrel{\left(\sigma_{X, \mathfrak{e}}\right)_{*} \tau_{\mathcal{F}}}{\longrightarrow}\left(\sigma_{X, \mathfrak{C}}\right)_{*} \mathcal{F}
$$

which by abuse of notation we also call $\tau_{\mathcal{F}}$. For every étale morphism $Y \rightarrow X$ it induces a homomorphism on global sections

$$
\tau_{\mathcal{F}}: \quad \Gamma\left(Y \times{ }_{L} \mathfrak{C}, \mathcal{F}\right) \longrightarrow \Gamma\left(Y \times_{L} \mathfrak{C},\left(\sigma_{X, \mathfrak{C}}\right)_{*} \mathcal{F}\right)=\Gamma\left(Y \times_{L} \mathfrak{C}, \mathcal{F}\right) .
$$

Definition 1.6. The étale sheaf on $X$ of $\tau$-invariants of $\underline{\mathcal{F}}$, denoted by $\underline{\mathcal{F}}^{\tau}$, is defined as the kernel of

$$
\left(\mathrm{id}-\tau_{\mathcal{F}}\right): W\left(p r_{*} \mathcal{F}\right) \longrightarrow W\left(p r_{*} \mathcal{F}\right),
$$


where $p r: X \times_{L} \mathfrak{C} \rightarrow X$ is the projection onto the first factor. I.e., for every étale morphism $Y \rightarrow X$ we have

$$
\underline{\mathcal{F}}^{\tau}(Y):=\left\{f \in \Gamma\left(Y \times_{L} \mathfrak{C}, \mathcal{F}\right): \tau_{\mathcal{F}}(f)=f\right\} .
$$

The sheaf $\underline{\mathcal{F}}^{\tau}$ is an étale sheaf of $\underline{A}_{X}$-modules (Section A.3). If $Y$ is connected and $\underline{\mathcal{F}}$ is locally free, we will see in Corollary 4.3 that the sections $\underline{\mathcal{F}}^{\tau}(Y)$ form a projective $A$-module of rank at most rk $\mathcal{F}$. To simplify our notation, in later sections we will usually abbreviate $W\left(p r_{*} \mathcal{F}\right)$ by $W(\mathcal{F})$.

$\tau$-invariants $I I$ :

Suppose $\underline{\mathcal{F}}$ is an algebraic $\tau$-sheaf over $A / \mathfrak{a}$ on $X$ for some non-zero ideal $\mathfrak{a}$ of $A$. As in the previous construction, one can associate to $\underline{\mathcal{F}}$ the étale sheaf $(\underline{\mathcal{F}} / \mathfrak{a} \underline{\mathcal{F}})^{\tau}$ of $\tau$-invariants modulo $\mathfrak{a}$, defined as the kernel of the induced homomorphism

$$
\left(\text { id }-\tau_{\mathcal{F}}\right): W\left(p r_{*} \mathcal{F}\right) \longrightarrow W\left(p r_{*} \mathcal{F}\right) \text {. }
$$

The sheaf $\underline{\mathcal{F}}^{\tau}$ is an étale sheaf of $\underline{A / \mathfrak{a}} X$-modules.

\section{TORSION POINTS}

Throughout this section let $X$ be a rigid analytic space, $\mathcal{F}$ be a locally free rigid analytic $\tau$-sheaf over $A(1)$ on $X$ of rank $r$, and $\mathfrak{a} \subsetneq A$ be a non-zero ideal. The $\tau$ sheaf $\underline{\mathcal{F}} / \mathfrak{a} \underline{\mathcal{F}}$ of construction III of change of coefficients is locally free on $X \times_{\mathbb{F}_{q}} A / \mathfrak{a}$. By abuse of notation, we denote its direct image on $X$ again by $\underline{\mathcal{F}} / \mathfrak{a} \mathcal{F}$. It is locally free of rank $r \cdot \operatorname{dim}_{\mathbb{F}_{q}} A / \mathfrak{a}$. On $X$, the homomorphism $\tau$ induces a homomorphism

$$
\sigma_{X}^{*}(\mathcal{F} / \mathfrak{a} \mathcal{F}) \longrightarrow \mathcal{F} / \mathfrak{a} \mathcal{F} \text {. }
$$

In later sections we will study the sheaf of global $\tau$-invariant sections of $\underline{\mathcal{F}}$, at least étale locally. Now any given section of $\underline{\mathcal{F}}^{\tau}$ will be $\mathfrak{p}$-adically approximated by a suitable compatible systems of sections of the sheaves $\left(\underline{\mathcal{F}} / \mathfrak{p}^{n} \underline{\mathcal{F}}\right)^{\tau}$. The former element may be thought of as an analytic solution of an algebraic equation, the latter as a formal solution to the same equation. The formal solutions will have a simpler structure, and in general there will be more such than analytic ones. In this section we will study formal solutions (and those only up to a finite level), as a preparation for the study of analytic solutions in the following sections.

Definition 2.1. $\quad$ (a) (cf. Taguchi, Wan [27, § 6] or Drinfeld [9, § 1]) The space of $\mathfrak{a}$-torsion points of $\underline{\mathcal{F}}$ is the rigid analytic group-space on $X$ given by

$$
\mathfrak{a} \underline{\mathcal{F}}:=\operatorname{Spec}_{X} \operatorname{Sym}_{\mathcal{O}_{X}}(\mathcal{F} / \mathfrak{a} \mathcal{F}) /(\text { Frob }-\tau)
$$

where the ideal $($ Frob $-\tau)$ is generated by the local sections $f^{q}-\tau f$ for $f \in \mathcal{F} / \mathfrak{a} \mathcal{F} \subset \operatorname{Sym}_{\mathcal{O}_{X}}(\mathcal{F} / \mathfrak{a} \mathcal{F})$. The structure of a commutative group is induced from the group structure of $\mathcal{F} / \mathfrak{a} \mathcal{F}$. The action of $A / \mathfrak{a}$ on $\mathcal{F} / \mathfrak{a} \mathcal{F}$ induces an action $A / \mathfrak{a} \rightarrow \operatorname{End}_{X}(\mathfrak{a} \underline{\mathcal{F}})$.

(b) The étale sheaf $\underline{\mathcal{F}}[\mathfrak{a}]$ on $X$ of $\mathfrak{a}$-torsion points of $\underline{\mathcal{F}}$ is the sheaf represented by a $\underline{\mathcal{F}}$, i.e., for $\pi: Y \rightarrow X$ étale we have

$$
\begin{aligned}
\underline{\mathcal{F}}[\mathfrak{a}](Y): & \operatorname{Hom}_{X}\left(Y,{ }_{\mathfrak{a}} \underline{\mathcal{F}}\right) \\
= & \left\{h \in \operatorname{Hom}_{\mathcal{O}_{Y}}\left(\pi^{*}(\mathcal{F} / \mathfrak{a} \mathcal{F}), \mathcal{O}_{Y}\right): h(\tau f)=h(f)^{q}\right. \\
& \left.\quad \text { for all local sections } f \text { of } \pi^{*}(\mathcal{F} / \mathfrak{a} \mathcal{F})\right\} .
\end{aligned}
$$

The actions of $A / \mathfrak{a}$ on $\mathfrak{a} \underline{\mathcal{F}}$ or on $\underline{\mathcal{F}} / \mathfrak{a} \underline{\mathcal{F}}$ induce the same $A / \mathfrak{a}$-module structures on $\underline{\mathcal{F}}[\mathfrak{a}]$. 
Remark 2.2. The group space $\mathfrak{a} \underline{\mathcal{F}}$ is finite étale over $X$ of degree $\#(A / \mathfrak{a})^{r}$. This may be verified locally on an admissible affinoid covering $\left\{\operatorname{Sp} B_{i}\right\}$ which trivializes $\mathcal{F} / \mathfrak{a} \mathcal{F}$. Let $f=\left(f_{1}, \ldots, f_{s}\right)$ be an $\mathbb{F}_{q}$-basis of $\mathcal{F} / \mathfrak{a} \mathcal{F}$ over Sp $B_{i}$ where $s=r \operatorname{dim}_{\mathbb{F}_{q}} A / \mathfrak{a}$. There is a matrix $\Delta \in \mathrm{GL}_{s}\left(B_{i}\right)$ such that $\tau f=f \Delta$. Then

$$
\mathfrak{a} \underline{\mathcal{F}} \times{ }_{X} \operatorname{Sp} B_{i}=\operatorname{Sp} B_{i}[f] /\left({ }^{\sigma} f-f \Delta\right)
$$

is finite étale over $\operatorname{Sp} B_{i}$.

In addition to these sheaves, we consider the constant sheaf $\mathcal{H o m}_{\mathbb{F}_{q}}\left(\underline{A / \mathfrak{a}}, \mathbb{F}_{q}\right)$ which is fiber-wise free over $A / \mathfrak{a}$ of rank one.

Definition 2.3. A pairing $\mathcal{G}_{0} \times \mathcal{G}_{1} \rightarrow \mathcal{H}$ of étale sheaves $\mathcal{G}_{0}, \mathcal{G}_{1}, \mathcal{H}$ of $A$ /a-modules on $X$ is called perfect if it induces isomorphisms of $A / \mathfrak{a}$-module sheaves $\mathcal{G}_{i} \cong$ $\mathcal{H o m}_{\underline{A} \mathbf{a}}\left(\mathcal{G}_{1-i}, \mathcal{H}\right)$.

Lemma 2.4. Suppose the morphism $\tau$ in (2.1) is bijective. Then

(a) There exists a locally finite étale covering $\pi: Y \rightarrow X$ such that $(\underline{\mathcal{F}} / \mathfrak{a} \underline{\mathcal{F}})^{\tau}(Y)$ is (on connected components) free over $A / \mathfrak{a}$ of rank $r$ and such that there is an isomorphism of coherent sheaves

$$
(\underline{\mathcal{F}} / \mathfrak{a} \underline{\mathcal{F}})^{\tau}(Y) \otimes_{\mathbb{F}_{q}} \mathcal{O}_{Y} \stackrel{\cong}{\longrightarrow} \pi^{*}(\mathcal{F} / \mathfrak{a} \mathcal{F}) .
$$

(b) The pairing

$$
\underline{\mathcal{F}}[\mathfrak{a}] \times(\underline{\mathcal{F}} / \mathfrak{a} \underline{\mathcal{F}})^{\tau} \longrightarrow \mathcal{H o m}_{\mathbb{F}_{q}-\operatorname{Mod}}\left(\underline{A / \mathfrak{a}}, \mathbb{F}_{q}\right), \quad(h, f) \longmapsto(a \mapsto h(a f))
$$

of étale sheaves of $A / \mathfrak{a}$-modules on $X$ is perfect.

Proof. (a) We follow closely Anderson [1, Lemma 1.8.2]. If $\tau$ is bijective on $\mathcal{F} / \mathfrak{a} \mathcal{F}$, then it is so on $\mathcal{F} / \mathfrak{a}^{n} \mathcal{F}$. Because some power of $\mathfrak{a}$ is principal, say equal to $(a)$, it suffices to treat this case. We then choose $\mathbb{F}_{q}[t] \subset A$ as the homomorphism defined by $t \mapsto a$. Using Remark 1.5 we also choose an admissible covering of $X$ by affinoid subdomains such that on each of these subdomains $\operatorname{Sp} B_{i}$ the sheaf $\mathcal{F}$ is associated to a free $B_{i}\langle t\rangle$-module. Then $(\mathcal{F} / \mathfrak{a} \mathcal{F})\left(\operatorname{Sp} B_{i}\right)$ is a free $B_{i}$-module of rank $s=r \cdot \operatorname{dim}_{\mathbb{F}_{q}} A / \mathfrak{a}$ on which $\tau$ acts as $\Delta \cdot \sigma$ for a matrix $\Delta \in \mathrm{GL}_{s}\left(B_{i}\right)$. We consider the equation

$$
\Phi=\Delta^{\sigma} \Phi \quad \text { and } \quad 1=\operatorname{det} \Delta \cdot(\operatorname{det} \Phi)^{q-1}
$$

for an $s \times s$-matrix $\Phi$. Let $Y_{i}:=\operatorname{Sp} B_{i}^{\prime}$ be the finite étale covering of $\operatorname{Sp} B_{i}$ obtained by adjoining the entries of $\Phi$ to $B_{i}$. Then the columns of the matrix $\Phi \in \mathrm{GL}_{s}\left(B_{i}^{\prime}\right)$ form a $B_{i}^{\prime}$-basis of $(\mathcal{F} / \mathfrak{a} \mathcal{F})\left(Y_{i}\right)$ as well as a $\mathbb{F}_{q}$-basis of $(\underline{\mathcal{F}} / \mathfrak{a} \mathcal{F})^{\tau}\left(Y_{i}\right)$. So on every connected component of $Y$ the $\mathbb{F}_{q^{-}}$-dimension of $(\underline{\mathcal{F}} / \mathfrak{a} \mathcal{F})^{\tau}\left(Y_{i}\right)$ is $r \operatorname{dim}_{\mathbb{F}_{q}} A / \mathfrak{a}$. Since by [1, Lemma 1.8.2] the stalks of $(\underline{\mathcal{F}} / \mathfrak{a} \underline{\mathcal{F}})^{\tau}$ are free $A / \mathfrak{a}$-modules of rank $r$, the proof of (a) is complete.

(b) The question is local on $X$, and so we may assume that the isomorphism (a) holds over $X$. Then

$$
\begin{aligned}
\underline{\mathcal{F}}[\mathfrak{a}](X) & =\left\{h \in \operatorname{Hom}_{\mathcal{O}_{X}}\left(\mathcal{F} / \mathfrak{a} \mathcal{F}, \mathcal{O}_{X}\right): h(\tau f)=h(f)^{q} \text { for all } f\right\} \\
& =\operatorname{Hom}_{\mathbb{F}_{q}(X)}\left((\underline{\mathcal{F}} / \mathfrak{a} \underline{\mathcal{F}})^{\tau}(X), \underline{\mathbb{F}_{q}}(X)\right) \\
& \cong \operatorname{Hom}_{\underline{A / \mathfrak{a}(X)}}\left((\underline{\mathcal{F} / \mathfrak{a} \mathcal{F}})^{\tau}(X), \operatorname{Hom}_{\mathbb{F}_{q}}\left(\underline{A / \mathfrak{a}}, \mathbb{F}_{q}\right)(X)\right),
\end{aligned}
$$


the last isomorphism mapping $h \in \underline{\mathcal{F}}[\mathfrak{a}](X)$ to $f \longmapsto(a \mapsto h(a f))$. The same chain of isomorphisms holds for all étale morphisms $Y \rightarrow X$, and so the pairing is perfect.

Theorem 2.5. Suppose that (2.1) is an isomorphism. Then there exists a (finite) étale $\mathrm{GL}_{r}(A / \mathfrak{a})$-torsor $\pi: X_{\mathcal{F}, \mathfrak{a}} \rightarrow X$ such that

(a) $\underline{\mathcal{F}}[\mathfrak{a}]\left(X_{\mathcal{F}, \mathfrak{a}}\right)$ is a free $A / \mathfrak{a}\left(X_{\mathcal{F}, \mathfrak{a}}\right)$-module of rank $r$ and

(b) $(\underline{\mathcal{F}} / \mathfrak{a} \underline{\mathcal{F}})^{\tau}\left(X_{\underline{\mathcal{F}}, \mathfrak{a}}\right)$ is a free $\overline{A / \mathfrak{a}}\left(X_{\underline{\mathcal{F}}, \mathfrak{a}}\right)$-module of rank $r$. Furthermore, the natural homomorphism of sheaves on $Y$

$$
(\underline{\mathcal{F}} / \mathfrak{a} \underline{\mathcal{F}})^{\tau}\left(X_{\underline{\mathcal{F}, \mathfrak{a}}}\right) \otimes_{\mathbb{F}_{q}} \mathcal{O}_{X_{\underline{\mathcal{F}}, \mathfrak{a}}} \stackrel{\sim}{\longrightarrow} \pi^{*} \mathcal{F} / \mathfrak{a} \mathcal{F}
$$

is an isomorphism.

Proof. (a) This follows from general principles on sections of finite étale group schemes; cf. Katz-Mazur [19, Chap. 1]. To be precise a $\mathcal{F}$ is a finite étale commutative group over $X$ of $\operatorname{rank}(\# A / \mathfrak{a})^{r}$. Thus the functor which assigns to every rigid analytic $X$-space $T$ the set

$$
\underline{\operatorname{Isom}}_{X-\mathrm{Gp}}\left((\underline{A / \mathfrak{a}})^{r}, \mathfrak{a} \underline{\mathcal{F}}\right)(T)=\left\{\text { group isomorphisms }(\underline{A / \mathfrak{a}})^{r}(T) \stackrel{\sim}{\longrightarrow} \mathfrak{a} \underline{\mathcal{F}}(T)\right\}
$$

is representable by a rigid analytic space $Y^{\prime}$, which is finite étale over $X$; cf. SGA 3 , Cor. X.5.10]. (To apply the theory written for schemes to rigid analytic spaces, cover $X$ by affinoids $\operatorname{Sp} B_{i}$ and work over $\operatorname{Spec} B_{i}$. The representing schemes $Y_{i}$ are finite étale over Spec $B_{i}$ and induce rigid analytic spaces which glue due to their universal property.) Therefore the open and closed sub-functor

$$
\begin{aligned}
& \underline{\operatorname{Isom}}_{A / \mathfrak{a}-\operatorname{Mod}}\left(\underline{A / \mathfrak{a}}^{r}, \mathfrak{a} \underline{\mathcal{F}}\right)(T) \\
&=\left\{A / \mathfrak{a}-\text { module isomorphisms }(\underline{A / \mathfrak{a}})^{r}(T) \stackrel{\sim}{\longrightarrow} \mathfrak{a} \underline{\mathcal{F}}(T)\right\} \\
& \subset \underline{\operatorname{Isom}}_{X-\mathrm{Gp}}\left((\underline{A / \mathfrak{a}})^{r},{ }_{\mathfrak{a}} \underline{\mathcal{F}}\right)(T)
\end{aligned}
$$

is representable by a rigid analytic space $Y \subset Y^{\prime}$, which is finite étale over $X$. Above every connected component of $X$ the space $Y$ is either empty or a $\mathrm{GL}_{r}(A / \mathfrak{a})$-torsor. Finally the surjectivity of $\pi: Y \rightarrow X$ follows from Lemma 2.4.

(b) We deduce from (a) and Lemma 2.4 that

$$
(\underline{\mathcal{F}} / \mathfrak{a} \underline{\mathcal{F}})^{\tau}\left(X_{\mathcal{\mathcal { F }}, \mathfrak{a}}\right) \cong \mathcal{H o m}_{\underline{A} / \mathfrak{a}}\left(\underline{\mathcal{F}}[\mathfrak{a}], \mathcal{H o m}_{\mathbb{F}_{q}}\left(\underline{A / \mathfrak{a}}, \mathbb{F}_{q}\right)\right)\left(X_{\underline{\mathcal{F}, \mathfrak{a}}}\right)
$$

is a free $\underline{A / \mathfrak{a}}\left(X_{\underline{\mathcal{F}, \mathfrak{a}}}\right)$-module of rank $r$. The last statement also follows from Lemma 2.4 .

\section{THE KEY-LEMMA FOR $\tau$-SHEAVES}

In this section we prove the following Key-Lemma on the extension of sections of a $\tau$-sheaf. We will apply the Key-Lemma to show that $\underline{\mathcal{F}}^{\tau}$ is an overconvergent sheaf (Definition A.12). In the following sections the Key-Lemma will serve as the main tool for studying the analytic triviality of $\tau$-sheaves. For the required background on analytic points see Appendix A.1.

Key-Lemma 3.1. Let $X=\operatorname{Sp} B$ be affinoid, set $\mathfrak{C}:=\mathfrak{A}(1)$ and let $\mathcal{F}$ be a rigid analytic $\tau$-sheaf over $L\langle t\rangle$ on $X$ such that $\mathcal{F}$ is a free $B\langle t\rangle$-module of rank $r$. Let $g_{1}, \ldots, g_{m} \in \Gamma\left(X \times_{L} \mathfrak{C}, \mathcal{F}\right)$. Further let $x \in \mathcal{M}(X)$ be an analytic point and let 
$\bar{f}_{1}, \ldots, \bar{f}_{m}$ be given elements of $\left.x^{*} \mathcal{F}\right)\left(k(x)^{\text {alg }}\right)$, defined over an algebraic closure of $k(x)$, which satisfy $\bar{f}_{i}-\tau \bar{f}_{i}=g_{i}(x)$ for all $i$. Then:

(a) There exists an étale morphism $\pi: V \rightarrow X$ of affinoids such that $\pi V$ is a wide neighborhood of $x$ in $X$, a point $y \in \mathcal{M}(V)$ above $x$, and uniquely determined sections $f_{1}, \ldots, f_{m} \in \Gamma\left(V \times_{L} \mathfrak{C}, \mathcal{F}\right)$ satisfying $f_{i}(y)=\bar{f}_{i}$ and $f_{i}-\tau f_{i}=\pi^{*} g_{i}$ for all $i$. Moreover,

(b) If $\tau$ is bijective on $\mathcal{F} / t \mathcal{F}$, then $V$ may be taken as an affinoid subdomain of $X_{\mathcal{F}, t^{N}}$, constructed in Theorem 2.5 (with the obvious morphism to $X$ ) for some $N \gg 0$.

(c) If all $\bar{f}_{i}$ are defined over $k(x)$, we can find $V \subset X$ as a wide affinoid neighborhood of $x$.

Proof. We have to find $m$ sections of $\mathcal{F}$, which we will represent by an $r \times m$ matrix $\Phi$, using a basis of $\mathcal{F}$ over $B\langle t\rangle$. We choose this basis such that $\sup _{n}\left|\Delta_{n}\right|=1$; cf. Remark 1.5. Let $\Omega=\sum_{n \geq 0} \Omega_{n} t^{n} \in M_{r \times m}(B\langle t\rangle)$ be the coordinate matrix of the system $\left(g_{1}, \ldots, g_{m}\right)$ with respect to this basis. The condition $f_{i}-\tau f_{i}=g_{i}$ translates into the system of equations

$$
\Phi_{n}-\Delta_{0}{ }^{\sigma} \Phi_{n}=\Psi_{n}:=\Omega_{n}+\sum_{\nu=1}^{n} \Delta_{\nu}{ }^{\sigma} \Phi_{n-\nu}, \quad n \geq 0 .
$$

For each of the matrices $\Phi_{n}$ this is an étale equation. Note that if $\left|\Psi_{n}\right|<1$, then there is a unique solution $\Phi_{n}$ with $\left|\Phi_{n}\right|=\left|\Psi_{n}\right|$, given explicitly by

$$
\Phi_{n}=\sum_{\nu=0}^{\infty} \Delta_{0}{ }^{\sigma} \Delta_{0} \cdot \ldots \cdot{ }^{\sigma^{\nu-1}} \Delta_{0}{ }^{\nu} \Psi_{n}
$$

Set $\left(\bar{f}_{1}, \ldots, \bar{f}_{m}\right)=\sum_{n \in \mathbb{N}_{0}} \bar{\Phi}_{n} t^{n}=: \bar{\Phi}$ with matrices $\bar{\Phi}_{n} \in M_{r \times m}(k(x))$.

If $x$ is a classical point and the $\bar{f}_{1}, \ldots, \bar{f}_{m}$ are defined over $k(x)$, then we can use the Inverse Function Theorem to extend $\bar{\Phi}$ to a wide neighborhood $U$ of $x$. Namely, we lift $\bar{\Phi}$ to an element of $M_{r \times m}(B\langle t\rangle)$ and set $\Phi=\bar{\Phi}+\Phi^{\prime}$. If $U$ is such that $\left|\Phi_{\nu}^{\prime}\right| \ll 1$ on $U$ for all $0 \leq \nu<n$, there is a unique solution $\Phi_{n}^{\prime}$ on $U$ with $\left|\Phi_{n}^{\prime}\right| \ll 1$ to equation (3.1) as in (3.2).

In the general case however, we cannot explicitly solve equation (3.1) for all $n$. Instead, we formally adjoin the solutions of the equations for $n=0, \ldots, N$, thus obtaining an étale morphism $V \rightarrow X$. On $V$ we now may solve equation (3.1) explicitly for all $n>N$ as in (3.2).

To be precise, we fix constants $\Theta$ and $\epsilon$ in $\left|L^{\text {alg }}\right|$ satisfying

$$
\begin{aligned}
& 0<\Theta<\frac{1}{2} \quad \text { and } \\
& 0<\epsilon<\inf \left\{1, \frac{1}{2} \Theta\left|\bar{\Phi}_{n}\right|^{-q}: n \geq 0\right\} \leq 1 .
\end{aligned}
$$

There is an $l \in \mathbb{N}$ such that

$$
\left|\Delta_{n}\right| \leq \epsilon, \quad\left|\bar{\Phi}_{n}\right| \leq \frac{1}{2} \Theta \quad \text { and } \quad\left|\Omega_{n}\right| \leq \frac{1}{2} \Theta \quad \text { for every } n>l \text {. }
$$

We set $N:=2 l$ and define $B_{N}$ to be the polynomial ring over $B$ in the components of the matrices $\Phi_{0}, \ldots, \Phi_{N}$ modulo the relations (3.1) for $n=0, \ldots, N$.

If $\tau$ is bijective on $\mathcal{F} / t \mathcal{F}$, the matrix $\Delta_{0}$ is invertible. In this case $B_{N}$ is an affinoid algebra and $\pi: Y:=\operatorname{Sp} B_{N} \rightarrow \operatorname{Sp} B$ is the finite étale $\mathrm{GL}_{r}\left(\mathbb{F}_{q}[t] / t^{N}\right)$-torsor 
above $\operatorname{Sp} B$ which trivializes the $t^{N+1}$-torsion of $\underline{\mathcal{F}}$; cf. Thm. 2.5. Now consider the affinoid subsets

$$
\begin{aligned}
& V:=\left\{y \in \operatorname{Sp} B_{N}:\left|\Phi_{n}(y)\right| \leq \Theta \quad \forall l<n \leq N \quad \text { and } \quad\left|\Phi_{n}(y)\right|^{q} \leq \Theta / \epsilon \quad \forall 0 \leq n \leq l\right\}, \\
& U:=\left\{y \in \operatorname{Sp} B_{N}:\left|\Phi_{n}(y)\right| \leq \frac{\Theta}{2} \quad \forall l<n \leq N \quad \text { and } \quad\left|\Phi_{n}(y)\right|^{q} \leq \frac{\Theta}{2 \epsilon} \quad \forall 0 \leq n \leq l\right\}
\end{aligned}
$$

of $\operatorname{Spec} B_{N}$.

The choice of our constants implies that $x$ lifts to an analytic point $y \in \mathcal{M}(U)$ with $\bar{\Phi}_{n}=\Phi_{n}(y)$ for all $n \leq N$. We claim that equation (3.1) possesses solutions $\Phi_{n}$ in $\Gamma\left(V, \mathcal{O}_{V}\right)$ for all $n>N$ which satisfy $\left|\Phi_{n}\right| \leq \Theta$. Indeed, by induction

$$
\left|\Psi_{n}\right| \leq \max \left\{\left|\Omega_{n}\right|,\left|\Delta_{\nu}\right|\left|\Phi_{n-\nu}\right|^{q}, 1 \leq \nu \leq n\right\}
$$

and for $n>N$ the right hand side is at most the maximum of $\left|\Omega_{n}\right| \leq \frac{1}{2} \Theta$ and

$$
\begin{aligned}
& \sup \left\{\left|\Delta_{n}\right|: n \geq 0\right\} \cdot \Theta^{q}=\Theta^{q} \leq \frac{1}{2} \Theta \quad \text { for } \quad \nu \leq l, \\
& \epsilon \cdot\left(\sup \left\{\left|\Phi_{n}\right|: n \geq 0\right\}\right)^{q} \leq \epsilon \Theta / \epsilon=\Theta \quad \text { for } \quad \nu>l .
\end{aligned}
$$

Hence we may take for $\Phi_{n}$ the solution to equation (3.1) given by (3.2). It is the unique solution with $\left|\Phi_{n}\right|=\left|\Psi_{n}\right| \leq \Theta$. Therefore we have $\bar{\Phi}_{n}=\Phi_{n}(y)$ also for all $n>N$. The same argument together with $\lim _{\nu \rightarrow \infty}\left|\Delta_{\nu}\right|=0$ and $\lim _{n \rightarrow \infty}\left|\Omega_{n}\right|=0$ shows that

$$
\limsup _{n \rightarrow \infty}\left|\Phi_{n}\right| \leq\left(\limsup _{n \rightarrow \infty}\left|\Phi_{n}\right|\right)^{q} \leq \Theta^{q}<1,
$$

hence $\lim _{n \rightarrow \infty}\left|\Phi_{n}\right|=0$. So the columns of

$$
\Phi:=\sum_{n=0}^{\infty} \Phi_{n} t^{n}
$$

are the desired elements $f_{1}, \ldots, f_{m}$ satisfying $f_{i}-\tau f_{i}=\pi^{*} g_{i}$. These are uniquely determined by the condition $f_{i}(y)=\bar{f}_{i}$, since the defining equation (3.1) for the $\Phi_{n}$ is étale.

Let $\widetilde{\Theta}, \widetilde{\epsilon} \in L^{\text {alg }}$ be constants with $|\widetilde{\Theta}|=\Theta$ and $|\widetilde{\epsilon}|=\epsilon$. Then the elements $\widetilde{\Theta}^{-1} \Phi_{n} \in B_{N}$ for $l<n \leq N$ and $(\widetilde{\epsilon} / \widetilde{\Theta})^{1 / q} \Phi_{n} \in B_{N}$ for $0 \leq n \leq l$ form an affinoid generating system of $V$ over $X$. Therefore we see that $U$ is relatively compact in $V$ over $X$, i.e., $U \subset \subset_{X} V$. Thus by [18, Lemma 3.4.2] the image $\pi(V)$ is a wide neighborhood of $\pi(U)$ in $X$. Since $x \in \pi \mathcal{M}(U)=\mathcal{M}(\pi U)$ by [18, Prop 3.1.7], we conclude that $\pi V$ is a wide neighborhood of $x$ in $X$.

If all $\bar{f}_{i}$ are defined over $k(x)$, we have $k(y)=k(x)$. By [18, Lemma 3.1.5] there is an affinoid subdomain $U^{\prime} \subset U$ with $y \in \mathcal{M}\left(U^{\prime}\right)$ such that $\left.\pi\right|_{U^{\prime}}$ is an isomorphism $U^{\prime} \rightarrow \pi\left(U^{\prime}\right)$. Now by [18, Lemma 3.4.2] there is a wide neighborhood $V^{\prime}$ of $y$ such that $\left.\pi\right|_{V^{\prime}}: V^{\prime} \rightarrow \pi V^{\prime}$ is an isomorphism and $\pi V^{\prime}$ is a wide neighborhood of $x$ in $X$, i.e., $\mathcal{M}\left(\pi V^{\prime}\right)$ is a neighborhood of $x$ in $\mathcal{M}(X)$.

Corollary 3.2. Let $\underline{\mathcal{F}}$ be a locally free rigid analytic $\tau$-sheaf over $A(1)$ on a connected $X$ and let $\pi: \bar{Y} \rightarrow X$ be a general morphism; cf. Definition A.9. Then the homomorphism of A-modules

$$
\underline{\mathcal{F}}^{\tau}(X) \longrightarrow\left(\pi^{*} \underline{\mathcal{F}}\right)^{\tau}(Y), \quad f \mapsto \pi^{*} f
$$

is injective and its image is a saturated A-submodule. 
Proof. For the proof, we may assume that $A=\mathbb{F}_{q}[t]$. Since $\underline{\mathcal{F}}^{\tau}$ is also a Zariski sheaf, we may assume that $X$ is an affinoid over which we have local coordinates for $\underline{\mathcal{F}}$ as in Remark 1.5 .

The proposition is clear in the case when $X$ and $Y$ are spectra of algebraically closed complete fields. Let $y$ be an analytic point of $Y$ and $x=\pi(y)$. We let $\widehat{k(x)^{a l} g}$ denote the completion of an algebraic closure of $k(x)$. By considering the diagram

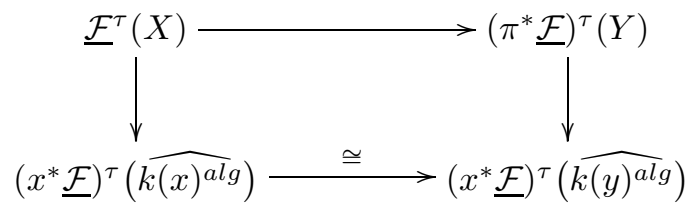

the assertion on injectivity is clear, if we have shown it for the vertical morphisms, i.e., it remains to consider the case $Y=\operatorname{Sp} \widehat{k(x)^{a l} g}$. In local coordinates the defining equation (3.1) for the coefficients of $\tau$-invariants are étale. Therefore if we have a global section which vanishes at $x$ (étale locally), it will have to vanish on the entire connected component of $X$ containing $x$. Since $X$ is connected, injectivity is established.

To prove saturation of $h$, again by the above diagram it suffices to consider the case where $Y=\operatorname{Sp} \widehat{k(x)^{a l} g}$. Now let $f$ be in $\underline{\mathcal{F}}^{\tau}(X), 0 \neq \bar{g}$ in $\left(x^{*} \underline{\mathcal{F}}\right)^{\tau}\left(\widehat{k(x)^{a l} g}\right)$, and $a \in A$ such that $f(x)=a \bar{g}$. We have to show that $\bar{g}$ extends to a section of $\underline{\mathcal{F}}^{\tau}$ over $X$. We may also assume that $a$ is not a constant, since then the assertion is clear.

By replacing $A$ by $\mathbb{F}_{q}[a]$, we may assume $a=t$. Let $\sum_{n} v_{n} t^{n}$ be the series representing $\bar{g}$ with coefficients in $\widehat{k(x)^{a l} g}$. Then $\sum v_{n} t^{n+1}$ represents $f$ and has coefficients in $\Gamma\left(X, \mathcal{O}_{X}\right)$. But then the $v_{n}$ are in $\Gamma\left(X, \mathcal{O}_{X}\right)$, and hence $\sum_{n} v_{n} t^{n}$ lies in $\underline{\mathcal{F}}^{\tau}(X)$ and maps to $\bar{g}$.

Corollary 3.3. Let $\underline{\mathcal{F}}$ be a locally free rigid analytic $\tau$-sheaf over $A(1)$. Then

(a) For every analytic point $x \in \mathcal{M}(X)$ the étale sheaves $x^{*}\left(\underline{\mathcal{F}}^{\tau}\right)$ and $\left(x^{*} \underline{\mathcal{F}}\right)^{\tau}$ on $\operatorname{Sp} k(x)$ are canonically isomorphic.

(b) For every étale analytic point $y \in \mathcal{M}_{\text {ét }}(X)$ (Section A.3), the stalk of $\underline{\mathcal{F}}^{\tau}$ at $y$ is given by $\left(y^{*} \underline{\mathcal{F}}\right)^{\tau}(k(y))$.

(c) $\underline{\mathcal{F}}^{\tau}$ is an overconvergent sheaf on the small étale site of $X$.

(d) The homomorphism (id $-\tau): W(\mathcal{F}) \rightarrow W(\mathcal{F})$ is surjective if and only if it is so for the fibers $y^{*} \mathcal{F}$ at all étale analytic points $y \in \mathcal{M}_{\text {ét }}(X)$.

Proof. All assertions are local. Thus by Lemma 1.4 and Remark 1.5 we may assume that $A=\mathbb{F}_{q}[t]$ and that $\mathcal{F}$ is free of finite rank on $X \times \mathfrak{A}(1)$. To see part (a), note that by the previous corollary the canonical morphism of étale sheaves $x^{*}\left(\underline{\mathcal{F}}^{\tau}\right) \rightarrow\left(x^{*} \underline{\mathcal{F}}\right)^{\tau}$ is injective. That it is surjective follows directly from Lemma 3.1 (a). Next, part (b) is just a reformulation of (a).

For (c) let $Y \rightarrow X$ be étale and $V \subset Y$ a special subset. We need to show that

$$
\underline{\mathcal{F}}^{\tau}(V)=\lim _{V \subset \subset_{Y} U} \underline{\mathcal{F}}^{\tau}(U)
$$

and so let $f \in \underline{\mathcal{F}}^{\tau}(V)$. By Lemma 3.1 (c) with $m=1$ and $g_{1}=0$, there exists for every analytic point $y$ of $V$ a wide affinoid neighborhood $U_{y}$ of $y$ in $Y$ and a unique section $\tilde{f}_{y} \in \underline{\mathcal{F}}^{\tau}\left(U_{y}\right)$ such that $f(y)=\widetilde{f}_{y}(y)$. By the compactness of $\mathcal{M}(V)$ finitely 
many of the $\mathcal{M}\left(U_{y}\right)$ will cover $\mathcal{M}(V)$. So there are finitely many $y$ such that the special subset $U:=\bigcup U_{y}$ is a wide neighborhood of $V$ in $Y$. Since the sections $\widetilde{f}_{y}$ agree with $f$ on $V$, by the previous corollary applied to suitable points in $V$ and to $Y$, they glue to a section $\widetilde{f} \in \underline{\mathcal{F}}^{\tau}(U)$.

Finally (d) is a direct consequence of the first assertion of the Key-Lemma 3.1 (a), since if a special set $X$ is covered by wide open sets, it is covered by finitely many of these.

Proposition 3.4. Let $\underline{\mathcal{G}}$ be a locally free $\tau$-sheaf over $A(\infty)$ on $X$, and let $\underline{\mathcal{F}}$ be the locally free $\tau$-sheaf over $A(1)$ on $X$, obtained from $\underline{\mathcal{G}}$ by the Change of coefficients II (Section 1.2). Then the natural homomorphism $\underline{\mathcal{G}}^{\tau} \rightarrow \underline{\mathcal{F}}^{\tau}$ is an isomorphism of étale sheaves of A-modules.

Proof. We may assume that $A=\mathbb{F}_{q}[t]$. Choose $c \in L$ such that $|c|>1$ and define $D_{m}:=\operatorname{Sp} L\left\langle\frac{t}{c^{m}}\right\rangle$, so that $X \times_{L} D_{m}, m \in \mathbb{N}$, is an admissible covering of $X \times_{L}(\operatorname{Spec} A)^{\mathrm{rig}}$. We have to show that $\left(\left.\underline{\mathcal{G}}\right|_{X \times_{L} D_{m}}\right)^{\tau} \rightarrow \underline{\mathcal{F}}^{\tau}$ is an isomorphism for all $m \geq 0$.

Since both sheaves are overconvergent by Corollary 3.3 , it suffices to verify the isomorphy on étale stalks, and hence to assume that $X=\operatorname{Sp} F$ for some algebraically closed complete normed field $F$ above $L$. The $\tau$-sheaf $\underline{\mathcal{G}}$ is thus given by a free finitely generated module $M$ over

$$
F\langle\langle t\rangle\rangle:=\left\{\sum_{\nu \geq 0} a_{\nu} t^{\nu}\left|a_{\nu} \in F ; \forall \rho>1: \lim _{\nu \rightarrow \infty}\right| a_{\nu} \mid \rho^{\nu}=0\right\}
$$

of some rank $r$, on which $\tau_{\mathcal{G}}$ is represented by a matrix $\Delta=\sum \Delta_{\nu} t^{\nu} \in M_{r}(F\langle\langle t\rangle\rangle)$, i.e.,

$$
\forall \rho>1: \lim _{\nu \rightarrow \infty}\left|\Delta_{\nu}\right| \rho^{\nu}=0 .
$$

The elements of $\underline{\mathcal{F}}^{\tau}(X)$ can be written as vectors $\Phi=\sum \Phi_{n} t^{n} \in F\langle t\rangle^{r}$, i.e., with

$$
\lim _{n \rightarrow \infty}\left|\Phi_{n}\right|=0
$$

and subject to the condition $\Phi=\Delta^{\sigma} \Phi$, i.e.,

$$
\Phi_{n}-\Delta_{0}{ }^{\sigma} \Phi_{n}=\Psi_{n}:=\sum_{\nu=1}^{n} \Delta_{\nu}{ }^{\sigma} \Phi_{n-\nu}, \quad n \geq 0 .
$$

We set $\rho:=c^{m+1}, m \in \mathbb{N}$, and claim that $\left|\Phi_{n}\right| \rho^{n}$ is bounded - note that this implies that $\left|\Phi_{n}\right|\left|c^{m}\right|^{n}$ converges to zero. As $m$ was arbitrary this will finish the proof. Now the sequences in (3.3) and (3.4) are zero sequences. Therefore we may find some $N \in \mathbb{N}$ such that for all $n>N$ and $\nu \in\{0, \ldots, n\}$ we have

$$
\left|\Delta_{\nu}\right| \rho^{\nu}\left|\Phi_{n-\nu}\right|^{q-1} \leq 1 / 2 .
$$

Now let $n>N$. For $\nu=0$ the inequality implies $\left|\Delta_{0}{ }^{\sigma} \Phi_{n}\right|<\left|\Phi_{n}\right|$, and therefore $\left|\Phi_{n}\right|=\left|\Psi_{n}\right|$. Hence

$$
\begin{aligned}
\left|\Phi_{n}\right| \rho^{n} & \leq \rho^{n} \max _{\nu=1, \ldots, n}\left|\Delta_{\nu}\right|\left|\Phi_{n-\nu}\right|^{q} \leq \max _{\nu=1, \ldots, n}\left(\left|\Delta_{\nu}\right| \rho^{\nu}\left|\Phi_{n-\nu}\right|^{q-1}\right)\left|\Phi_{n-\nu}\right| \rho^{n-\nu} \\
& \leq 1 / 2 \max _{\nu=0, \ldots, n-1}\left|\Phi_{\nu}\right| \rho^{\nu},
\end{aligned}
$$

which shows that $\left|\Phi_{n}\right| \rho^{n}$ is bounded and thus proves the claim. 


\section{Triviality OF $\tau$-ShEAVES}

The simplest $\tau$-sheaf is $\underline{\mathbb{1}}_{X, A(1)}$. This section is centered around the question of how to recognize whether a given (locally free) rigid analytic $\tau$-sheaf is (basically) of this trivial form. In the following section, we study the same question étale locally which leads to the notion of analytic triviality.

Definition 4.1. A (locally free) rigid analytic $\tau$-sheaf $\underline{\mathcal{F}}$ on $X$ is trivial if there exists a finitely generated projective $A$-module $P$ such that $\underline{\mathcal{F}} \cong P \otimes_{A} \underline{\mathbb{1}}_{X, A(1)}$.

If $\underline{\mathcal{F}}$ is trivial, then clearly $P \cong \underline{\mathcal{F}}^{\tau}(X)$.

Let us first rephrase some results of Anderson, [1, § 2], in our language.

Lemma 4.2. Suppose $L^{\prime}$ is a finite field extension of $L$ and $X=\operatorname{Sp} L^{\prime}$.

(a) Any sub- $\tau$-sheaf of a trivial $\tau$-sheaf on $X$ is trivial.

(b) Suppose $\underline{\mathcal{F}}$ is a locally free rigid analytic $\tau$-sheaf over $A(1)$ on $X$ with module of global $\tau$-invariants $P:=\underline{\mathcal{F}}^{\tau}(X)$. Then $P$ is finitely generated projective over $A$ and the induced homomorphism

$$
h: P \otimes_{A} \underline{\mathbb{1}}_{X, A(1)} \rightarrow \underline{\mathcal{F}}
$$

is injective. In particular the rank of $P$ over $A$ is bounded by the rank of $\underline{\mathcal{F}}$ over $X \times \mathfrak{A}(1)$.

(c) If either the image of $h$ in (b) is of full rank, or equivalently (by (b)), if $\underline{\mathcal{F}}$ and $P$ are of the same rank, then $h$ is an isomorphism.

Proof. To prove (a), suppose $\underline{\mathcal{F}}=P \otimes_{A} \underline{\mathbb{1}}_{X, A(1)}$ is trivial, suppose $\underline{\mathcal{G}} \subset \underline{\mathcal{F}}$ is a $\tau$-subsheaf and set $P:=\underline{\mathcal{F}}^{\tau}(X)$ and $Q:=\underline{\mathcal{G}}^{\tau}(X)$. It will suffice to show that $Q \otimes_{A} \underline{\mathbb{1}}_{X, A(1)} \rightarrow \underline{\mathcal{G}}$ is an isomorphism. To prove this, we may assume that $A=\mathbb{F}_{q}[t]$. Let $\underline{\mathcal{F}^{\prime \prime}}$ be the cokernel of $\underline{\mathcal{G}} \hookrightarrow \underline{\mathcal{F}}$. By Anderson [1, Lemma 2.10.4] there is a finite field extension $L^{\prime \prime}$ of $L^{\prime}$ such that

$$
\underline{\mathcal{G}} \otimes_{L^{\prime}} L^{\prime \prime} \cong\left(\underline{\mathcal{G}} \otimes_{L^{\prime}} L^{\prime \prime}\right)^{\tau}\left(L^{\prime \prime}\right) \otimes_{A} \underline{\mathbb{1}}_{\operatorname{Sp} L^{\prime \prime}, A(t)} .
$$

Because the functor of $\tau$-invariants is left exact and because of Corollary 3.2, we have the commutative diagram

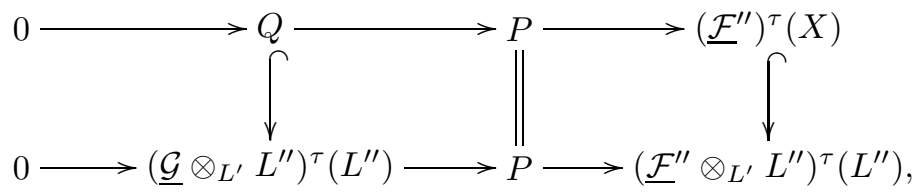

in which the rows are left exact and the vertical homomorphisms are injective. Since $\underline{\mathcal{F}}^{\prime \prime}$ is not necessarily locally free, strictly speaking Corollary 3.2 does not apply. But we may apply it to the right hand term in $0 \rightarrow \underline{\mathcal{F}}_{\text {tors }}^{\prime \prime} \rightarrow \underline{\mathcal{F}}^{\prime \prime} \rightarrow \underline{\mathcal{F}}^{\prime \prime} / \underline{\mathcal{F}}_{\text {tors }}^{\prime \prime}$, and on the left the injectivity assertion is clear from Section 2 .

A simple diagram chase implies that $Q \hookrightarrow\left(\underline{\mathcal{G}} \otimes_{L^{\prime}} L^{\prime \prime}\right)^{\tau}\left(L^{\prime \prime}\right)$ must be an isomorphism, and so in particular the rank of $Q$ over $A$ agrees with the rank of $\mathcal{G}$ over $X \times \mathfrak{A}(1)$. Therefore $Q \otimes_{A} \underline{\mathbb{1}}_{X, A(1)} \hookrightarrow \underline{\mathcal{G}}$ is a $\tau$-subsheaf of full rank. Since after base change along the faithfully flat morphism $L^{\prime} \rightarrow L^{\prime \prime}$ it becomes an isomorphism, it must have been an isomorphism to begin with. This proves (a).

To prove (b), we may replace $\underline{\mathcal{F}}$ by the image of $h$, since the latter is a locally free $\tau$-subsheaf of $\underline{\mathcal{F}}$ and since it has the same $\tau$-invariants on $X$ as $\underline{\mathcal{F}}$. Considering the ranks of the domain and range of $h$, we then find a projective $A$-submodule $P^{\prime}$ of $P$ 
of rank equal to the rank of $\underline{\mathcal{F}}$. Let $\underline{\mathcal{G}}$ denote the kernel of $\underline{\mathcal{F}}^{\prime}:=P^{\prime} \otimes_{A} \underline{\mathbb{1}}_{X, A(1)} \rightarrow \underline{\mathcal{F}}$. Since taking $\tau$-invariants is left exact, the definition of $h$ implies $\underline{\mathcal{G}}^{\tau}(X)=0$. By part (a) it follows that $\underline{\mathcal{G}}=0$, and hence $h$ is injective on $\underline{\mathcal{F}}^{\prime}$. Therefore the cokernel of $h: \underline{\mathcal{F}}^{\prime} \rightarrow \underline{\mathcal{F}}$ must be of finite length over $X \times{ }_{L} \mathfrak{A}(1)$. Taking $\tau$-invariants yields

$$
0 \longrightarrow P^{\prime} \longrightarrow P \longrightarrow\left(\underline{\mathcal{F}}^{\prime \prime}\right)^{\tau} \text {. }
$$

As before, the term on the right is finite, and this completes the proof of (b).

For part (c), it remains to prove the surjectivity of $h$. We may again assume $A=\mathbb{F}_{q}[t]$. Then $P$ is free, and so we may choose an $A$-basis $f_{1}, \ldots, f_{r}$ of it. We now use that $k(x)\langle t\rangle$ is a principal ideal domain, as is shown for instance in 1, $\S 2]$, and denote by $\alpha_{\nu}$ the elementary divisors of a matrix representing $h$. If we apply the $r$-th exterior power operation to $h$, the resulting elementary divisor is $\alpha_{1} \cdot \ldots \cdot \alpha_{r}$. Because $\tau$ preserves the image, the product ${ }^{\sigma} \alpha_{1} \cdot \ldots \cdot{ }^{\sigma} \alpha_{r}$ divides $\alpha_{1} \cdot \ldots \cdot \alpha_{r}$, and thus all $\alpha_{\nu}$ lie in $\mathbb{F}_{q}[t] \cdot k(x)\langle t\rangle^{\times}$. It follows that there is an $a \in \mathbb{F}_{q}[t]$ such that $a \mathcal{F}$ is a $\tau$-submodule of $\operatorname{im}(h) \cong P \otimes_{A} L^{\prime}\langle t\rangle$. By part (a) we must have $a \underline{\mathcal{F}} \cong(a \underline{\mathcal{F}})^{\tau}(X) \otimes_{A} \underline{\mathbb{1}}_{X, A(1)}$, and (c) is now immediate.

Corollary 4.3. Suppose $X$ is connected and $\underline{\mathcal{F}}$ is a locally free rigid analytic $\tau$ sheaf over $A(1)$ on $X$. Then $P:=\underline{\mathcal{F}}^{\tau}(X)$ is a finitely generated projective A-module of rank at most the rank of $\mathcal{F}$. Equality of ranks holds if and only if $\mathcal{F}$ is trivial.

Proof. By Corollary 3.2, the first assertion may be reduced to the case where $X$ is the spectrum of $k(x)$ with $x$ a classical point on $X$. However this case is proved in the previous lemma. It remains to show that

$$
h: \underline{\mathbb{1}}_{X, A(1)} \otimes_{A} \underline{\mathcal{F}}^{\tau}(X) \longrightarrow \underline{\mathcal{F}}, \quad b \otimes f \mapsto b f
$$

is an isomorphism of rigid analytic $\tau$-sheaves on $X \times{ }_{L} \mathfrak{A}(1)$ provided that the ranks of $\mathcal{F}$ and $P$ agree, since the converse implication is obvious.

Because domain and range of $h$ are locally free of the same rank, it suffices to prove surjectivity on fibers at all classical points $x$. In other words, if $P_{x}$ denotes the global sections of $\left(x^{*} \underline{\mathcal{F}}\right)^{\tau}$, we have to prove (i) that for any such $x$ the inclusion $P \hookrightarrow P_{x}$ is an equality and (ii) that $P_{x} \otimes_{A} \underline{\mathbb{1}}_{x, A(1)} \longrightarrow x^{*} \mathcal{F}$ is an isomorphism. By our assumption on ranks and part (b) of the previous lemma, the ranks of $P$, of $P_{x}$ and of $x^{*} \underline{\mathcal{F}}$ agree. Part (c) of the lemma then establishes (ii). Since $P$ is saturated in $P_{x}$ by Corollary 3.2 , having the same ranks implies (i).

Remark 4.4. If $\underline{\mathcal{F}}$ arises by coefficient change from a $\tau$-sheaf $\left(\mathcal{G}, \tau_{\mathcal{G}}\right)$ over $A(\infty)$, we have seen in Proposition 3.4 that $\underline{\mathcal{F}}^{\tau}(X) \cong \underline{\mathcal{G}}^{\tau}(X)$. So in particular $\operatorname{rk}_{A} \underline{\mathcal{F}}^{\tau}(X)=$ $\operatorname{rk}_{A} \underline{\mathcal{G}}^{\tau}(X)$. However, it is in general not true that

$$
\left(\mathcal{G}, \tau_{\mathcal{G}}\right) \cong \underline{\mathbb{1}}_{X, A(\infty)} \otimes_{A} \underline{\mathcal{G}}^{\tau}(X)
$$

even if $\operatorname{rk}_{A} \underline{\mathcal{G}}^{\tau}(X)=\operatorname{rk} \mathcal{G}$. The reason for this is that in cases we will be interested in later, the homomorphism $\tau_{\mathcal{G}}$ is typically not an isomorphism over all of $\mathfrak{A}(\infty)$. Then the support $D$ of the coherent sheaf

$$
\mathcal{G} /\left(\underline{\mathbb{1}}_{X, A(\infty)} \otimes_{A} \underline{\mathcal{G}}^{\tau}(X)\right)
$$

is discrete in $X \times{ }_{L} \mathfrak{A}(\infty)$ and satisfies $\sigma_{X, \mathfrak{A}(\infty)}^{*}(D) \subset D$. Hence $D$ has a limit point at $\infty$. On the other hand since $\mathfrak{A}(1)$ is compact there may be no limit points of $D$ on $X \times{ }_{L} \mathfrak{A}(1)$. So $\tau_{\mathcal{F}}$ must be an isomorphism over $\mathfrak{A}(1)$. 


\section{Analytic triviality of $\tau$-Sheaves}

We now study analytic triviality which is the locally étale generalisation of triviality of the previous section, and which in fact we define 'on étale stalks'. After describing some basic properties, we will show that the locus of analytic triviality in $\mathcal{M}(X)$ is (Berkovich) open and give a first simple example. Using the openness property we show that any analytically trivial $\tau$-sheaf will be trivial over some temperate étale covering (Definition A.14).

Let $\underline{\mathcal{F}}$ be a locally free rigid analytic $\tau$-sheaf over $A(1)$ on $X$. In order to take a more general approach to triviality of a $\tau$-sheaf we will consider $\underline{\mathcal{F}}$ over the étale site. Generalising [1, (2.3)] and motivated by Corollary 4.3, we define:

Definition 5.1. The $\tau$-sheaf $\mathcal{F}$ is called analytically trivial if for every étale analytic point $y \in \mathcal{M}_{\text {ét }}(X)$ (Section A.3) the rank of $\left(y^{*} \underline{\mathcal{F}}\right)^{\tau}(k(y))$ as an $A$-module is equal to the rank of the sheaf $y^{*} \mathcal{F}$.

Clearly Definition 5.1 is in accordance with the usual principle that properties of étale sheaves can be defined and established on stalks at étale points. Note however that for general sheaves on rigid analytic spaces this principle is false. Theorem 5.10 below will show that indeed the above is a sensible definition.

We begin with two simple consequences of the above definition.

Proposition 5.2. If $\underline{\mathcal{F}}$ is analytically trivial, then so is $\pi^{*} \mathcal{F}$ for every general morphism $\pi: X^{\prime} \rightarrow \bar{X}$. Moreover the $\tau$-sheaves $\underline{\mathcal{F}}{ }^{\otimes n}, \operatorname{Sym}^{k} \underline{\mathcal{F}}$ and $\bigwedge^{n} \underline{\mathcal{F}}$ are analytically trivial.

We omit the obvious proof.

Proposition 5.3. Suppose $0 \rightarrow \underline{\mathcal{F}} \rightarrow \underline{\mathcal{G}} \rightarrow \underline{\mathcal{H}} \rightarrow 0$ is a short exact sequence of locally free rigid analytic $\tau$-sheaves. Then $\underline{\mathcal{G}}$ is analytically trivial if and only if the same holds for $\underline{\mathcal{F}}$ and $\underline{\mathcal{H}}$.

Proof. We only need to prove the assertion for $X=\operatorname{Sp} F$, where $F$ is a complete normed algebraically closed field. This is shown in [1, Lem. 2.7.2].

Next we investigate the analytically trivial locus of some $\underline{\mathcal{F}}$ on $X$.

Lemma 5.4. Suppose $\underline{\mathcal{F}}$ is a rigid analytic locally free $\tau$-sheaf of rank $r$ over $A(1)$ on $X$ such that $x^{*} \mathcal{F}$ is analytically trivial for some $x \in \mathcal{M}(X)$. Then there exists a wide open neighborhood $U$ of $x$ in $X$ such that $\underline{\mathcal{F}}$ is analytically trivial on $U$.

Note that if for some $x \in \mathcal{M}(X)$ there exists a general morphism from a point $y$ to $x$ at which $y^{*} \mathcal{F}$ is analytically trivial, then by the definition of analytic triviality $x^{*} \underline{\mathcal{F}}$ is analytically trivial.

Proof. As usual, we may assume $A=\mathbb{F}_{q}[t]$. By our hypothesis there exists a finite separable field extension $L^{\prime}$ of $k(x)$ and elements $\bar{f}_{1}, \ldots, \bar{f}_{r} \in\left(x^{*} \underline{\mathcal{F}}\right)^{\tau}\left(L^{\prime}\right)$, which form an $\mathbb{F}_{q}[t]$-basis of $\left(x^{*} \underline{\mathcal{F}}\right)^{\tau}$. So by Lemma 3.1] there exist an étale morphism $\pi: Y \rightarrow X$, a point $y \in Y$, a wide open neighborhood $V$ of $y$ such that $L^{\prime} \cong k(y)$, the sections $\bar{f}_{j}$ extend to $V$ and $x=\pi(y)$. By Corollary 3.2 the rank of $\left(\pi^{*} \underline{\mathcal{F}}\right)^{\tau}(V)$ is again $r$, and so by Corollary 4.3, $\pi^{*} \mathcal{F}$ is analytically trivial on $V$. Moreover $U:=\pi(V)$ is a wide open neighborhood of $x$, and it follows that $\underline{\mathcal{F}}$ is analytically trivial on $U$. 
Theorem 5.5. Let $X$ be an affinoid rigid analytic space and $\underline{\mathcal{F}}$ a locally free rigid analytic $\tau$-sheaf over $A(1)$ on $X$. Then the subset

$$
M:=\left\{x \in \mathcal{M}(X): x^{*} \underline{\mathcal{F}} \text { is analytically trivial }\right\} \subset \mathcal{M}(X)
$$

is open.

Proof. This is immediate from the previous corollary. By it we have for each $x \in M$ a wide neighborhood $U$ of $x$ on which $\mathcal{F}$ is analytically trivial. This however means that $y^{*} \underline{\mathcal{F}}$ is analytically trivial at all $y \in \mathcal{M}(U)$, and the latter set is open in $\mathcal{M}(X)$.

Remark 5.6. By the same argument Theorem 5.5 can be proved more generally for paracompact rigid analytic spaces $X$; cf. the remark at the end of Section A.1

Example 5.9 and the example of Section 7 below raise the following question which goes back to a conversation with Pink:

Question 5.7. Let the notation be as in Theorem 5.5, Is the set $\mathcal{M}(X) \backslash M$ a special subset of $X$ ?

If the answer is in the affirmative, this would have strong consequences. For instance, since any non-empty special subset contains a classical point, a $\tau$-sheaf would be analytically trivial, if it is so at all classical points.

With respect to the canonical topology on the affinoid $L$-space $X$ the map $X \rightarrow$ $\mathcal{M}(X)$ is a homeomorphism onto a dense subset. Thus we see:

Corollary 5.8. Let $X$ be an affinoid rigid analytic space and $\underline{\mathcal{F}}$ a locally free rigid analytic $\tau$-sheaf over $A(1)$ on $X$. Then the subset

$$
U:=\left\{x \in X: x^{*} \mathcal{F} \text { is analytically trivial }\right\} \subset X
$$

is open with respect to the canonical topology on $X$.

To illustrate this situation we give a simple example. A more involved one will be computed in Section 7

Example 5.9. Let $A=\mathbb{F}_{q}[t], B=L\langle a, b\rangle$ and $X=\operatorname{Sp} B$. Consider the rigid analytic $\tau$-sheaf $\underline{\mathcal{F}}$ over $A(1)$ on $X$ given by the $B\langle t\rangle$-module $F=B\langle t\rangle$ and $\tau=$ $(a+b t) \cdot \sigma$. Then the subset of $\mathcal{M}(X)$ over which $\underline{\mathcal{F}}$ is analytically trivial is

$$
\{x \in \mathcal{M}(X):|b(x)|<|a(x)|\} .
$$

Proof. Fix an analytic point $x \in \mathcal{M}(X)$. We want to find a non-zero $\tau$-invariant $f$ which we write as $f=\sum_{n \geq 0} u_{n} t^{n}$, i.e., the coefficients have to satisfy

$$
u_{n}-a(x) u_{n}^{q}=b(x) u_{n-1}^{q} \quad \text { for all } \quad n \geq 0 .
$$

If $a(x)=0$, then $u_{n}=0$ for all $n$ and $\mathcal{F}$ is not analytically trivial at $x$.

So let $a(x) \neq 0$ and fix a $(q-1)^{\text {st }}$ root $\alpha \in k(x)^{a l g}$ of $a(x)$. Then setting $v_{n}=\alpha u_{n}$ the condition on the coefficients becomes

$$
v_{n}-v_{n}^{q}=c v_{n-1}^{q}, \quad c:=\frac{b(x)}{a(x)} .
$$

We may assume that $v_{0} \in \mathbb{F}_{q}^{\times}$.

If $|b(x)| \geq|a(x)|$, then by induction $\left|v_{n}\right|=|c|^{n / q} \geq 1$ and $\underline{\mathcal{F}}$ is not analytically trivial at $x$. 
If $|b(x)|<|a(x)|$, then we may choose $\left|v_{n}\right|=\left|c v_{n-1}^{q}\right|$ and by induction $\left|v_{n}\right|=$ $|c|^{1+q+\ldots+q^{n-1}}<1$. Hence $\sum_{n} v_{n} t^{n} \in k(x)\langle t\rangle$ and $\underline{\mathcal{F}}$ is analytically trivial at $x$. This finishes the proof.

Let $U$ be the admissible subset of $X$ on which $|b|<|a|$ and let $\pi: Y \rightarrow U$ be the finite étale Galois covering given by adjoining a $(q-1)^{\text {st }}$ root of $a$. Then $\mathrm{rk}_{A} \underline{\mathcal{F}}^{\tau}(Y)=1$, since we may explicitly solve equation (5.1) globally on $Y$. Thus we have $\pi^{*} \underline{\mathcal{F}} \cong \underline{\mathbb{1}}_{Y, A(1)} \otimes_{A}\left(\pi^{*} \underline{\mathcal{F}}\right)^{\tau}(Y)$.

Let us now give some equivalent conditions for analytic triviality:

Theorem 5.10. Let $X$ be an affinoid rigid analytic space and $\underline{\mathcal{F}}$ a locally free rigid analytic $\tau$-sheaf over $A(1)$ on $X$. Then the following are equivalent:

(a) $\mathcal{F}$ is analytically trivial,

(b) for every analytic point $x \in \mathcal{M}(X)$ the $\tau$-sheaf $x^{*} \mathcal{F}$ is analytically trivial,

(c) there exists a finite cover $U_{i}$ of $X$ by affinoids and étale morphisms $\pi_{i}$ : $V_{i} \rightarrow X$ with $\pi_{i}\left(V_{i}\right)=U_{i}$, such that the $\underline{\mathcal{F}}_{i}:=\pi_{i}^{*} \mathcal{F}$ satisfy

$$
\left(\underline{\mathcal{F}}_{i}\right)^{\tau}\left(V_{i}\right) \otimes_{A} \underline{\mathbb{1}}_{V_{i}, A(1)} \cong \underline{\mathcal{F}}_{i} .
$$

(d) there exists a connected temperate étale Galois covering $\pi: Z \rightarrow X$ with

$$
\pi^{*} \underline{\mathcal{F}} \cong \underline{\mathbb{1}}_{Z, A(1)} \otimes_{A} \underline{\mathcal{F}}^{\tau}(Z) .
$$

We do not know whether one can in fact trivialize an analytically trivial $\tau$ sheaf over a finite étale covering. The following example shows that for general (paracompact) rigid analytic spaces, this can (obviously) not be expected.

Example 5.11. Let $\underline{\mathcal{F}}$ be the $\tau$-sheaf associated to the universal Drinfeld- $A$-module of the analytic moduli space $\mathfrak{M}_{\mathfrak{n}}^{r}$ of Drinfeld- $A$-modules of some fixed rank $r$ and with some level $\mathfrak{n}$-structure; cf. 9]. Let $\Omega^{r}$ be the Drinfeld upper half space as in Examples 1.3 and let $\pi: \Omega^{r} \rightarrow \mathfrak{M}_{\mathfrak{n}}^{r}$ be the canonical covering constructed in 9 . Then $\pi^{*} \underline{\mathcal{F}}$ is in fact 'trivial' in the sense of Corollary 4.3. In particular $\underline{\mathcal{F}}$ is analytically trivial. However it may easily be shown that there does not exist a finite étale cover of $\mathfrak{M}_{\mathfrak{n}}^{r}$ over which $\underline{\mathcal{F}}$ becomes trivial.

Proof of Theorem 5.10. The equivalence $(\mathrm{a}) \Leftrightarrow(\mathrm{b})$ is clear from the definition and Corollary 3.3 (a) and and (b). The implication (c) $\Rightarrow(b)$ is clear from the definitions, and $(\mathrm{d}) \Rightarrow(\mathrm{c})$ follows from the fact that $Z \rightarrow X$ is a covering for the étale topology. We now prove $(\mathrm{b}) \Rightarrow(\mathrm{d})$.

By Remark 1.5 we may assume that $A=\mathbb{F}_{q}[t]$. As in the proof of Lemma 5.4, for $x \in \mathcal{M}(X)$ there exist an étale morphism $\pi_{x}: Y_{x} \rightarrow X$, a point $y \in Y_{x}$ with $\pi_{x}(y)=x$ and a wide open neighborhood $V_{x}$ of $y$ in $Y_{x}$ such that

$$
\left.\pi_{x}^{*} \underline{\mathcal{F}}\right|_{V_{x}} \cong\left(\pi_{x}^{*} \mathcal{\mathcal { F }}\right)^{\tau}\left(V_{x}\right) \otimes_{A} \underline{\mathbb{1}}_{V_{x}, A(1)} .
$$

Moreover by Lemma 3.1 (b), we may assume that $\pi_{x}$ is the morphism $X_{\mathcal{\mathcal { F } , t ^ { N _ { x } }}} \rightarrow X$ for some $N_{x} \in \mathbb{N}$.

By Proposition A.7 the $\pi\left(V_{x}\right)$ form an admissible covering of $X$. Since $X$ is quasi-compact, there is a finite set $S$ of analytic points $x$ such that the $\pi\left(V_{x}\right)$ for $x \in S$ form an admissible covering of $X$. Let

$$
N=\max \left\{N_{x}: x \in S\right\} .
$$


We set $Y=X_{\mathcal{F}_{,} t^{N}}$. Then $Y \rightarrow X$ is a torsor for the group $G=\mathrm{GL}_{r}\left(\mathbb{F}_{q}[t] / t^{N+1}\right)$. We replace $V_{x}$ by its preimage under the finite étale morphism $Y \rightarrow X_{\underline{\mathcal{F}}, t^{N}}$. Consider the admissible covering

$$
\mathcal{V}:=\left\{g V_{x}: x \in S, g \in G\right\}
$$

of $Y$. On $g V_{x}$ we have $\underline{\mathcal{F}}^{\tau}\left(g V_{x}\right) \cong\left(g^{-1}\right)^{*} \underline{\mathcal{F}}^{\tau}\left(V_{x}\right) \cong \underline{\mathcal{F}}^{\tau}\left(V_{x}\right) \cong \mathbb{F}_{q}[t]^{r}$. These isomorphisms form a Čech-cocycle

$$
\alpha \in \check{\mathrm{H}}^{1}\left(\mathcal{V}, \mathrm{GL}_{r}\left(\mathbb{F}_{q}[t]\right)\right) .
$$

In general one cannot expect to trivialize this cocycle by a finite étale covering of $Y$. However, it can be trivialized by a topological covering $Z$ of $Y$ which is Galois over $X$. We will construct $Z$. Let $N(\mathcal{V})$ be the nerve of the covering $\mathcal{V}$ of $Y$. Recall that this is a simplicial set whose $n$-simplices correspond to the connected components of the intersections of $n$ distinct elements of $\mathcal{V}$. There exists an equivalence of categories

$\{$ topological covering spaces of $Y$ split by $\mathcal{V}\} \cong\{$ covering spaces of $N(\mathcal{V})\}$.

Now let $\tilde{N}$ be the universal covering of $N(\mathcal{V})$ and let $Z$ be the corresponding topological covering space of $Y$. Clearly $Z$ is Galois over $Y$ with Galois group $\operatorname{Aut}(Z / Y)=\operatorname{Aut}(\widetilde{N} / N(\mathcal{V}))$. Furthermore, $Z \rightarrow X$ is a temperate étale Galois covering. Indeed, as $Z$ is universal for topological covering spaces of $Y$ split over $\mathcal{V}$, every $g \in G=\operatorname{Aut}(Y / X)$ lifts to an automorphism of $Z$. Therefore we have an exact sequence of discrete groups

$$
1 \rightarrow \operatorname{Aut}(Z / Y) \rightarrow \operatorname{Aut}(Z / X) \rightarrow \operatorname{Aut}(Y / X) \rightarrow 1
$$

From $Z / \operatorname{Aut}(Z / Y)=Y$ and $Y / \operatorname{Aut}(Y / X)=X$ we conclude that $Z / \operatorname{Aut}(Z / X)=$ $X$.

Moreover, the cocycle $\alpha$ is trivialized by $Z$ and hence $\underline{\mathcal{F}}^{\tau}(Z) \cong \mathbb{F}_{q}[t]^{r}$. (We may assume that $Z$ is connected by replacing $Y$ by one of its connected components.) Now the assertion follows with Corollary 4.3 .

Using for instance the characterization in part (b), the following is immediate, since it holds point-wise:

Corollary 5.12. If $\underline{\mathcal{F}}$ is analytically trivial, then $\tau_{\mathcal{F}}$ is an isomorphism.

\section{Exactness of the fundamental SEQUence}

In this section, we give some results on the right exactness of the Artin-Schreier type sequence (0.1) for $A$-coefficients from the introduction. Consider Example 5.9 and let $x \in \mathcal{M}(X)$ be an analytic point. The reader may easily verify that the sequence (0.1) for the fiber $x^{*} \underline{\mathcal{F}}$ over $x$ is right exact either if $x^{*} \underline{\mathcal{F}}$ is analytically trivial or if $\tau=0$. We will shortly see that this is a typical phenomenon.

Proposition 6.1. Suppose $\underline{\mathcal{F}}$ is analytically trivial over $A(1)$ on $X$. Then the sequence $0 \rightarrow \underline{\mathcal{F}}^{\tau} \rightarrow W(\mathcal{F}) \stackrel{1-\tau}{\rightarrow} W(\mathcal{F}) \rightarrow 0$ of étale sheaves is exact.

Proof. By Corollary 3.3 (d), it suffices to prove the assertion on fibers at étale analytic points. So let $F$ be a complete normed algebraically closed field extension of $L$. As $\underline{\mathcal{F}^{\tau}}$ is defined to be the kernel of id $-\tau$, the only part that needs proof is the surjectivity of $(1-\tau)$. It clearly suffices to consider the case where $\underline{\mathcal{F}}=\underline{\mathbb{1}}_{X, A}$. 
Let $f=\sum_{n=0}^{\infty} b_{n} t^{n} \in F\langle t\rangle$ be a section of $\mathcal{O}_{X \times{ }_{L} \mathfrak{A}(1)}$. We expand the equation $\left(1-\sigma_{X, \mathfrak{A}(1)}\right) g=f$ into powers of $t$ :

$$
(\mathrm{id}-\sigma)\left(\sum_{n=0}^{\infty} c_{n} t^{n}\right)=\sum_{n=0}^{\infty} b_{n} t^{n}
$$

where $g=\sum_{n=0}^{\infty} c_{n} t^{n}$. Since $F$ is algebraically closed, the resulting Artin-Schreier equations $c_{n}^{q}-c_{n}=b_{n}$ can all be solved inside $F$, and the condition $\left|b_{n}\right| \rightarrow 0$ for $n \rightarrow \infty$, implies the same for $\left|c_{n}\right|$. Hence $g$ lies in $F\langle t\rangle$, and the surjectivity is established.

Our next aim is to generalize the above. We consider $\tau$-sheaves over $A(1)$ on $X$.

Lemma 6.2. Any extension of an analytically trivial by a locally free nilpotent $\tau$-sheaf splits.

Proof. Consider a short exact sequence

$$
0 \longrightarrow \underline{\mathcal{F}} \longrightarrow \underline{\mathcal{E}} \longrightarrow \underline{\mathcal{G}} \longrightarrow 0
$$

in which $\underline{\mathcal{F}}$ is nilpotent, say $\tau_{\mathcal{F}}^{n}=0$, and locally free, and in which $\underline{\mathcal{G}}$ is analytically trivial. Because all the above sheaves are therefore locally free, the top sequence in the following commutative diagram is exact:

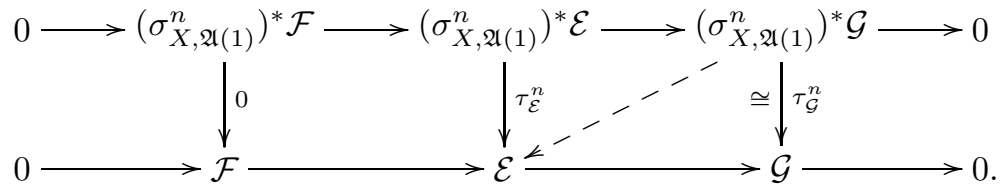

It follows that there is a unique dashed homomorphism. Its composite with the inverse of the isomorphism $\tau_{\mathcal{G}}^{n}$ is easily seen to be a splitting.

Corollary 6.3. If $\underline{\mathcal{F}}$ is a rigid analytic $\tau$-sheaf over $A(1)$ on $X$ with a filtration such that all subquotients are locally free and either nilpotent or analytically trivial. Then $\underline{\mathcal{F}}$ is an extension of a locally free nilpotent $\tau$-sheaf by an analytically trivial $\tau$-sheaf.

Proof. This is immediate from Proposition 5.3 and the previous lemma.

Theorem 6.4. Let $X$ be a rigid analytic space and $\underline{\mathcal{F}}$ a locally free rigid analytic $\tau$-sheaf over $A(1)$ on $X$. Assume that for every analytic point $x$ of $X$ the $\tau$-sheaf $x^{*} \underline{\mathcal{F}}$ is an extension of a locally free nilpotent by an analytically trivial $\tau$-sheaf. Then

$$
0 \longrightarrow \underline{\mathcal{F}}^{\tau} \longrightarrow W(\mathcal{F}) \stackrel{\text { id }-\tau_{\mathcal{F}}}{\longrightarrow} W(\mathcal{F}) \longrightarrow 0
$$

is an exact sequence of étale sheaves on $X$.

Proof. As $\underline{\mathcal{F}}^{\tau}$ is defined to be the kernel of id $-\tau_{\mathcal{F}}$, we only have to show that id $-\tau_{\mathcal{F}}$ is surjective. By Corollary 3.3 (d), it suffices to prove surjectivity on fibers at étale analytic points. Suppose therefore that $X=\operatorname{Sp} F$ for some complete normed algebraically closed field.

By assumption $\underline{\mathcal{F}}$ is an extension of a nilpotent locally free by an analytically trivial sheaf. By an obvious 9-term square, it suffices to prove surjectivity separately 
for the latter two cases. If $\underline{\mathcal{F}}$ is nilpotent, surjectivity is clear. In the other case, it was shown in Proposition 6.1.

Question 6.5. We pose the following problem which in spirit is similar to Question 5.7 and the remark following it: Suppose one assumes in the above theorem that $x^{*} \mathcal{F}$ is an extension of a locally free nilpotent by an analytically trivial $\tau$-sheaf only at every classical point $x$ of $X$. Does the conclusion of the theorem still hold?

Another interesting question is to give conditions under which one can recover $\underline{\mathcal{F}}$ from the locally étale sections of $\underline{\mathcal{F}}^{\tau}-$ at least up to nilpotent parts of $\underline{\mathcal{F}}$.

\section{An example}

The following example is due to R. Pink.

Let $\zeta \in K_{\infty}$ satisfy $|\zeta|<1$ and let

$$
X=\operatorname{Spec} K_{\infty}[a, b, c, d] /\left(a+d+2 \zeta, a d-b c-\zeta^{2}\right) .
$$

We set

$$
\Delta_{1}=\left(\begin{array}{ll}
a & b \\
c & d
\end{array}\right)
$$

and $\Delta=\operatorname{Id}+t \Delta_{1}$. Then $\operatorname{det} \Delta=(1-\zeta t)^{2}$. Let $X^{\text {rig }}$ be the analytification of $X$. As in the remark at the end of Section A.1 we consider analytic points $x$ of $X^{\text {rig }}$, and we represent them by their corresponding matrices $\Delta(x)$ and $\Delta_{1}(x)$.

On $X$ we define a (locally) free algebraic $\tau$-sheaf $\underline{\mathcal{F}}$ over $A=\mathbb{F}_{q}[t]$ by

$$
\mathcal{F}=\mathcal{O}_{X \times_{\mathbb{F}_{q}} \operatorname{Spec} A}^{\oplus 2}, \quad \tau=\Delta \cdot \sigma .
$$

There is an action of $\mathrm{GL}_{2}\left(\mathbb{F}_{q}\right)$ on $X$ and $X^{\text {rig }}$ given by

$$
\mathrm{GL}_{2}\left(\mathbb{F}_{q}\right) \times X \longrightarrow X, \quad(g, \Delta) \mapsto g \Delta g^{-1} .
$$

Under this action we have isomorphisms $g^{*} \underline{\mathcal{F}} \cong \underline{\mathcal{F}}$ for all $g \in \mathrm{GL}_{2}\left(\mathbb{F}_{q}\right)$. We denote the associated rigid analytic $\tau$-sheaf over $A(1)$ by $\underline{\mathcal{F}}$. Note that $\underline{\mathcal{F}}$ is the $\tau$-sheaf associated to the family of $\mathbb{F}_{q}[t]$-modules $(E, \phi)$ of dimension 2 and rank 2 with $E=\mathcal{O}_{X}^{2}$ and

$$
\phi_{t}=-\Delta_{1}^{-1}+\Delta_{1}^{-1} \sigma,
$$

when we take $\zeta^{-1}$ as the image of $t$ in $K_{\infty}$; cf. Section 8 . Moreover $X$ together with $\underline{\mathcal{F}}$ is a fine moduli space for "polarized" $t$-motives of rank 2 and dimension 2 with appropriate level structure; cf. [16, $\S 4]$.

The following result illustrates Theorem 5.5 .

\section{Proposition 7.1.}

$$
\begin{aligned}
\left\{y \in \mathcal{M}\left(X^{\mathrm{rig}}\right)\right. & \left.: y^{*} \widetilde{\mathcal{F}} \text { is analytically trivial }\right\} \\
& =\bigcup_{g \in \mathrm{GL}_{2}\left(\mathbb{F}_{q}\right)} g\left\{y \in \mathcal{M}\left(X^{\mathrm{rig}}\right):|a(y)|,|c(y)|,|d(y)|<1\right\} .
\end{aligned}
$$

Another explicit family of $t$-motives for Theorem 5.5 had been given by F. Gardeyn [12, II.2] in his thesis. His example can be considered as a special case of the above. 
Proof. We investigate the $\tau$-invariants of $\underline{\widetilde{\mathcal{F}}}$ which we write as $2 \times 2$-matrices $\Phi=$ $\Delta^{\sigma} \Phi$ with $\Phi=\sum_{n \geq 0} \Phi_{n} t^{n}$. We set $\Phi_{n}=\left(\begin{array}{ll}u_{n} & v_{n} \\ w_{n} & x_{n}\end{array}\right)$ and $\Phi_{0}=$ Id. This implies for $n \geq 1$

$$
\begin{aligned}
\Phi_{n}-{ }^{\sigma} \Phi_{n} & =\Delta_{1}{ }^{\sigma} \Phi_{n-1} \quad \text { or equivalently } \\
u_{n}-u_{n}^{q} & =a u_{n-1}^{q}+b w_{n-1}^{q} \\
w_{n}-w_{n}^{q} & =c u_{n-1}^{q}+d w_{n-1}^{q} \\
v_{n}-v_{n}^{q} & =a v_{n-1}^{q}+b x_{n-1}^{q} \\
x_{n}-x_{n}^{q} & =c v_{n-1}^{q}+d x_{n-1}^{q}
\end{aligned}
$$

The $\tau$-sheaf $y^{*} \underline{\mathcal{F}}$ is analytically trivial if and only if we can find solutions $\Phi_{n}(y)$ in $M_{2}\left(k(y)^{\text {alg }}\right)$ to these equations with $\left|\Phi_{n}(y)\right| \rightarrow 0$ for $n \rightarrow \infty$. Fix a point $y$ of $\mathcal{M}\left(X^{\mathrm{rig}}\right)$. To ease notation we write $a$ instead of $a(y)$, etc.

We first prove the inclusion " $\supset$ ". If $\left|\Delta_{1}\right|<1$, then the product

$$
\Phi:=\Delta \cdot{ }^{\sigma} \Delta \cdot \sigma^{2} \Delta \cdot \ldots
$$

converges in $\mathrm{GL}_{2}(k(y)\langle t\rangle)$ and hence $\underline{\tilde{\mathcal{F}}}$ is analytically trivial at $y$.

Next observe that $\underline{\mathcal{F}}$ is analytically trivial at $y$ if and only if it is at $g(y)$ for every $g \in \mathrm{GL}_{2}\left(\mathbb{F}_{q}\right)$. So it suffices to treat the following case.

Let $r \in K_{\infty}^{a l g}$ be a constant with $|\zeta| \leq|r|<1$ such that $|a|,|d| \leq|r|$ and $|r| \leq|b|$. This implies in particular

$$
|c|=\frac{|b c|}{|b|}=\frac{\left|a d-\zeta^{2}\right|}{|b|} \leq \frac{|r|^{2}}{|b|} \leq|r|<1 .
$$

Suppose we choose the solutions of (7.1) in such a way that

$$
\begin{aligned}
& \left|u_{n}-u_{n}^{q}\right|<1 \quad \Rightarrow \quad u_{n}|=| u_{n}-u_{n}^{q} \mid, \\
& \left|v_{n}-v_{n}^{q}\right|<1 \quad \Rightarrow \quad v_{n}|=| v_{n}-v_{n}^{q} \mid, \\
& \left|w_{n}-w_{n}^{q}\right|<1 \quad \Rightarrow \quad w_{n}|=| w_{n}-w_{n}^{q} \mid, \\
& \left|x_{n}-x_{n}^{q}\right|<1 \quad \Rightarrow \quad x_{n}|=| x_{n}-x_{n}^{q} \mid,
\end{aligned}
$$

for $n \geq 1$. Note that this is possible, because for $\alpha \in k(y)^{\text {alg }}$ with $|\alpha|<1$, the equation $x-x^{q}=\alpha$ has the solution $x=\sum_{\nu=0}^{\infty} \alpha^{q^{\nu}}$. In this situation we have the estimates

$$
\begin{aligned}
& \left|u_{n}\right|^{q} \leq \max \left\{|a|\left|u_{n-1}\right|^{q},|b|\left|w_{n-1}\right|^{q}\right\}, \\
& \left|w_{n}\right|^{q} \leq \max \left\{|c|\left|u_{n-1}\right|^{q},|d|\left|w_{n-1}\right|^{q}\right\}, \\
& \left|v_{n}\right|^{q} \leq \max \left\{|a|\left|v_{n-1}\right|^{q},|b|\left|x_{n-1}\right|^{q}\right\}, \\
& \left|x_{n}\right|^{q} \leq \max \left\{|c|\left|v_{n-1}\right|^{q},|d|\left|x_{n-1}\right|^{q}\right\} .
\end{aligned}
$$

These estimates are crude because we are not taking advantage of the gain we get when the right side is of absolute value less than one. From this we obtain the even 
cruder estimates

$$
\begin{aligned}
\left|u_{n}\right|^{q} & \leq \max \left\{|r|\left|u_{n-1}\right|^{q},|b|\left|w_{n-1}\right|^{q}\right\} \\
& \leq|r| \max \left\{\left|u_{n-1}\right|^{q},|b / r|\left|w_{n-1}\right|^{q}\right\}, \\
|b / r|\left|w_{n}\right|^{q} & \leq|b / r| \max \left\{\left|r^{2} / b\right|\left|u_{n-1}\right|^{q},|r|\left|w_{n-1}\right|^{q}\right\} \\
& \leq|r| \max \left\{\left|u_{n-1}\right|^{q},|b / r|\left|w_{n-1}\right|^{q}\right\} .
\end{aligned}
$$

These yield

$$
\max \left\{\left|u_{n}\right|^{q},|b / r|\left|w_{n}\right|^{q}\right\} \leq|r|^{n} \max \left\{\left|u_{0}\right|^{q},|b / r|\left|w_{0}\right|^{q}\right\},
$$

so that $\left|u_{n}\right| \rightarrow 0$ and $\left|w_{n}\right| \rightarrow 0$ as $n \rightarrow \infty$. A similar estimate shows that $\left|v_{n}\right| \rightarrow 0$ and $\left|x_{n}\right| \rightarrow 0$. We conclude that $y^{*} \underline{\mathcal{F}}$ is analytically trivial. This finishes the proof of the inclusion " $\supset$ ". (In Remark 7.2 we explain how this example also illustrates Theorem 5.10])

For the opposite inclusion fix an analytic point $y$ of $X^{\text {rig }}$ which does not belong to the right hand side of the claimed equality in Proposition 7.1 . Thus $|a|=|d| \geq 1$. We shall show that $y^{*} \underline{\widetilde{\mathcal{F}}}$ is not analytically trivial. Let us introduce the following new variables

$$
\widetilde{\Delta}_{1}=\left(\begin{array}{cc}
\tilde{a} & \tilde{b} \\
\tilde{c} & \tilde{d}
\end{array}\right):=\left(\begin{array}{cc}
-\zeta-a & -b \\
-c & -\zeta-d
\end{array}\right)=-\zeta \mathrm{Id}-\Delta_{1} .
$$

We have the relations

$$
\widetilde{\Delta}_{1}^{2}=0, \quad \tilde{b} \widetilde{\Delta}_{1}=\left(\begin{array}{cc}
-\tilde{b} \tilde{d} & \tilde{b}^{2} \\
-\tilde{d}^{2} & \tilde{b} \tilde{d}
\end{array}\right)=\left(\begin{array}{c}
\tilde{b} \\
\tilde{d}
\end{array}\right)\left(\begin{array}{ll}
-\tilde{d} & \tilde{b}
\end{array}\right) .
$$

Observe furthermore that $|a|=|d| \geq 1$ implies $|\tilde{a}|=|a|,|\tilde{b}|=|b|,|\tilde{c}|=|c|$ and $|\tilde{d}|=|d|$.

Let us now assume that $y$ is chosen such that the value $|\tilde{d}(y)|$ is minimal among all $|\tilde{d}(g(y))|$ for $g \in \mathrm{GL}_{2}\left(\mathbb{F}_{q}\right)$ and such that $|\tilde{b}(y)| \geq|\tilde{c}(y)|$. In particular we have $|\tilde{b}| \geq|\tilde{d}|=|\tilde{a}| \geq 1$. Note that the minimality assumption for $|\tilde{d}|$ implies

$$
\left|\tilde{b}^{q-1}-\tilde{d}^{q-1}\right|=\left|\tilde{b}^{q-1}\right| .
$$

Indeed if not, then writing $\tilde{b}^{q-1}-\tilde{d}^{q-1}=\prod_{\alpha \in \mathbb{F}_{q}^{\times}}(\tilde{b}-\alpha \tilde{d})$ yields an $\alpha \in \mathbb{F}_{q}^{\times}$with $|\tilde{b}-\alpha \tilde{d}|<|\tilde{b}|$. In particular $|\tilde{b}|=|\tilde{d}|$. But then

$$
\left(\begin{array}{cc}
1 & 0 \\
-\alpha^{-1} & 1
\end{array}\right) \widetilde{\Delta}_{1}\left(\begin{array}{cc}
1 & 0 \\
\alpha^{-1} & 1
\end{array}\right)=\left(\begin{array}{cc}
* & * \\
* & \tilde{d}-\alpha^{-1} \tilde{b}
\end{array}\right) .
$$

This contradicts the minimality of $|\tilde{d}|$.

Next one shows by induction that

$$
\begin{aligned}
\left(\begin{array}{c}
v_{n}^{q}-v_{n} \\
x_{n}^{q}-x_{n}
\end{array}\right) & =-\Delta_{1}\left(\begin{array}{c}
v_{n-1}^{q} \\
x_{n-1}^{q}
\end{array}\right) \\
& =-\Delta_{1}\left(\begin{array}{c}
v_{n-1}^{q}-v_{n-1} \\
x_{n-1}^{q}-x_{n-1}
\end{array}\right)-\Delta_{1}\left(\begin{array}{c}
v_{n-1} \\
x_{n-1}
\end{array}\right) \\
& =\sum_{j=1}^{n}\left(-\Delta_{1}\right)^{j}\left(\begin{array}{c}
v_{n-j} \\
x_{n-j}
\end{array}\right) .
\end{aligned}
$$


We have $\left(-\Delta_{1}\right)^{j}=\left(\zeta+\widetilde{\Delta}_{1}\right)^{j}=\zeta^{j}+j \zeta^{j-1} \widetilde{\Delta}_{1}$. Setting $y_{n}:=\left(\begin{array}{ll}-\tilde{d} & \tilde{b}\end{array}\right)\left(\begin{array}{c}v_{n} \\ x_{n}\end{array}\right)$ we can write

$$
\begin{aligned}
&\left(\begin{array}{c}
v_{n}^{q}-v_{n} \\
x_{n}^{q}-x_{n}
\end{array}\right)=\left(\begin{array}{c}
1 \\
\tilde{d} / \tilde{b}
\end{array}\right) y_{n-1} \\
&+\zeta\left(\begin{array}{c}
v_{n-1} \\
x_{n-1}
\end{array}\right)+\sum_{j=2}^{n}\left(j \zeta^{j-1}\left(\begin{array}{c}
1 \\
\tilde{d} / \tilde{b}
\end{array}\right) y_{n-j}+\zeta^{j}\left(\begin{array}{c}
v_{n-j} \\
x_{n-j}
\end{array}\right)\right) .
\end{aligned}
$$

We will see that the terms in the second line have smaller absolute values than the corresponding term in the first line. More precisely we make the following

Claim: For all $n \geq 0$ we have

$$
\begin{aligned}
& \left|x_{n}\right|=|\tilde{d}|^{\left(1-q^{-n}\right) /(q-1)} \geq\left|x_{n-1}\right| \geq 1, \\
& \left|v_{n}\right| \leq|\tilde{b}|\left|x_{n}\right|, \\
& \left|y_{n}\right|=|\tilde{b}|\left|x_{n}\right| .
\end{aligned}
$$

From the claim it follows that $\sum_{n} \Phi_{n} t^{n}$ does not converge for $|t|=1$ and that therefore $x^{*} \underline{\tilde{\mathcal{F}}}$ is not analytically trivial.

We prove the claim by induction on $n$. For $n=0$ we have $x_{0}=1, v_{0}=0, y_{0}=\tilde{b}$. So now assume the claim for $n-1$. From (7.4) we obtain

$$
\left|v_{n}^{q}-v_{n}\right|=|\tilde{b}|\left|x_{n-1}\right| \geq 1 \quad \text { and } \quad\left|x_{n}^{q}-x_{n}\right|=|\tilde{d}|\left|x_{n-1}\right| \geq 1 .
$$

This implies the claimed estimate for $\left|x_{n}\right|$ and the estimate

$$
\left|v_{n}\right|=|\tilde{b} / \tilde{d}|^{1 / q}\left|x_{n}\right| \leq|\tilde{b}|\left|x_{n}\right| .
$$

It remains to determine the absolute value of $y_{n}=\tilde{b} x_{n}-\tilde{d} v_{n}$. Multiplying (7.4) with $\left(\begin{array}{ll}-\tilde{d} & \tilde{b}\end{array}\right)$ from the left yields $\tilde{b} x_{n}^{q}-\tilde{d} v_{n}^{q}=\sum_{j=0}^{n} \zeta^{j} y_{n-j}$. Together with the equation $y_{n}^{q}=\tilde{b}^{q} x_{n}^{q}-\tilde{d}^{q} v_{n}^{q}$ we obtain:

$$
\left(\begin{array}{cc}
-\tilde{d}^{q} & \tilde{b}^{q} \\
-\tilde{d} & \tilde{b}
\end{array}\right) \cdot\left(\begin{array}{c}
v_{n}^{q} \\
x_{n}^{q}
\end{array}\right)=\left(\begin{array}{c}
y_{n}^{q} \\
\sum_{j=0}^{n} \zeta^{j} y_{n-j}
\end{array}\right) .
$$

By (7.3) the determinant of the $2 \times 2$-matrix is of absolute value $|\tilde{b}|^{q}|\tilde{d}|$. Solving for $v_{n}^{q}$, we find:

$$
\left|y_{n-1}\right|=|\tilde{b}|\left|x_{n-1}\right|=\left|v_{n}^{q}\right| \leq \max \left\{\frac{|\tilde{b}|}{|\tilde{d}|}\left(\frac{\left|y_{n}\right|}{|\tilde{b}|}\right)^{q}, \frac{\left|y_{n}\right|}{|\tilde{d}|}, \frac{|\zeta|^{1}\left|y_{n-1}\right|}{|\tilde{d}|}, \ldots, \frac{|\zeta|^{n}\left|y_{0}\right|}{|\tilde{d}|}\right\} .
$$

As $|\tilde{d}| \geq 1,|\zeta|<1$, and $\left|y_{j}\right|$ is increasing for $j=0, \ldots, n-1$, we either have

$$
\left|y_{n}\right| \geq\left|x_{n-1}\right||\tilde{b}||\tilde{d}| \quad \text { or } \quad \frac{\left|y_{n}\right|}{|\tilde{b}|} \geq\left(|\tilde{d}|\left|x_{n-1}\right|\right)^{1 / q}=\left|x_{n}\right| .
$$

Since $|\tilde{d}| \geq 1$, the formula $\left|x_{n}\right|=|\tilde{d}|^{\left(1-q^{-n}\right) /(q-1)}$ gives $\left|x_{n-1}\right||\tilde{d}| \geq\left|x_{n}\right|$, so that we have shown $\left|y_{n}\right| \geq\left|x_{n}\right||\tilde{b}|$. The converse inequality is clear from

$\left|y_{n}\right|=\left|\tilde{b} x_{n}-\tilde{d} v_{n}\right| \leq \max \left\{|\tilde{b}|\left|x_{n}\right|,|\tilde{d}|\left|v_{n}\right|\right\} \leq \max \left\{|\tilde{b}|\left|x_{n}\right|,|\tilde{b} / \tilde{d}|^{1 / q}|\tilde{d}|\left|x_{n}\right|\right\}=|\tilde{b}|\left|x_{n}\right|$.

This concludes the proof of Proposition 7.1 . 
Remark 7.2. We want to explain the relation to Theorem 5.10. Consider constants $r, s \in K_{\infty}^{a l g}$ with $|\zeta| \leq|r|<1<|s|$ and the affinoid subdomain of $X^{\text {rig }}$

$$
\operatorname{Sp} B=\left\{y \in X^{\text {rig }}:|a(y)|,|c(y)|,|d(y)| \leq|r|,|r| \leq|b(y)| \leq|s|\right\} .
$$

We have just shown that for every analytic point $y \in \mathcal{M}(B)$ the $\tau$-sheaf $y^{*} \underline{\widetilde{\mathcal{F}}}$ is analytically trivial.

Let $N \in \mathbb{Z}$ be minimal with $\left|r^{N} s\right|<1$ and define the finite étale $B$-algebra $B^{\prime}$ as

$$
B\left\langle\frac{v_{1}}{s^{1 / q}}, \frac{v_{2}}{(r s)^{1 / q}}, \ldots, \frac{v_{N}}{\left(r^{N-1} s\right)^{1 / q}}\right\rangle
$$

modulo the relations

$$
v_{1}-v_{1}^{q}=b, \quad v_{n}-v_{n}^{q}=a v_{n-1}^{q}+b x_{n-1}^{q}, \quad x_{n}=\sum_{\nu=0}^{\infty}\left(c v_{n-1}^{q}+d x_{n-1}^{q}\right)^{q^{\nu}} .
$$

Note that the series defining $x_{n}$ converges in $B^{\prime}$ by the estimates below. Namely, the estimates from (7.2) when applying the initial values for $\Phi_{0}$ (and analogous estimates for the $\left|x_{n}\right|$ and $\left.\left|v_{n}\right|\right)$ imply:

$$
\max \left\{\left|u_{n}\right|^{q},|b / r|\left|w_{n}\right|^{q}\right\} \leq|r|^{n} \quad \text { and } \max \left\{\left|v_{n}\right|^{q},|b / r|\left|x_{n}\right|^{q}\right\} \leq|r|^{n-1}|b| .
$$

Thus $\left|u_{n}\right|,\left|w_{n}\right|,\left|x_{n}\right| \leq|r|^{n / q}$ and $\left|v_{n}\right| \leq\left(|s||r|^{(n-1)}\right)^{1 / q}$. Because $|d|,|c|<1$, a simple inductive argument proves the convergence of the series for $x_{n}$. From this we also see that $B^{\prime}$ is indeed finite over $B$. Moreover these estimates imply that the series $\Phi=\sum_{n \geq 0} \Phi_{n} t^{n}$ converges in $M_{2}\left(B^{\prime}\langle t\rangle\right)$. So the columns of $\Phi$ generate an $A$-submodule of $\underline{\widetilde{\mathcal{F}}}^{\tau}\left(\mathrm{Sp} B^{\prime}\right)$ of rank 2. The morphism $\operatorname{Sp} B^{\prime} \rightarrow \operatorname{Sp} B$ is a Galois covering with Galois group

$$
G=\left\{g=\left(\begin{array}{cc}
1 & \sum_{n=1}^{N} g_{n} t^{n} \\
0 & 1
\end{array}\right)\right\} \mathrm{GL}_{2}\left(\mathbb{F}_{q}[t]\right)
$$

operating on $B^{\prime}$ via

$$
g\left(\begin{array}{l}
v_{n} \\
x_{n}
\end{array}\right)=\left(\begin{array}{l}
v_{n} \\
x_{n}
\end{array}\right)+\sum_{i=1}^{n} g_{i}\left(\begin{array}{c}
u_{n-i} \\
w_{n-i}
\end{array}\right) .
$$

So in this example the temperate étale Galois covering of $\operatorname{Sp} B$ from Theorem 5.10 can be chosen finite. However, we do not know whether this is true in general.

\section{Families of Abelian $A$-modules}

8.1. Definitions. Let $X$ be a rigid analytic space over $L$. We introduce a category of rigid analytic vector group spaces over $X$. Namely let $\operatorname{Vec}(X)$ be the category of locally free coherent $\mathcal{O}_{X}$-modules (by the usual abuse of language called vector bundles) with $\mathbb{F}_{q}$-linear algebraic group homomorphisms $\alpha: E \rightarrow E^{\prime}$, i.e., locally with respect to an admissible affinoid covering $\operatorname{Sp} B_{i}, i \in I$, of $X$ the morphism $\alpha$ can be given by a polynomial

$$
\sum_{\nu=0}^{m_{i}} A_{i, \nu} \sigma^{\nu}: B_{i}^{n} \longrightarrow B_{i}^{n^{\prime}}
$$

where $A_{i, \nu} \in M_{n, n^{\prime}}\left(B_{i}\right)$, the restrictions of $E$ and $E^{\prime}$ to $\operatorname{Sp} B_{i}$ are free of rank $n$ and $n^{\prime}$, respectively, and $\sigma$ denotes the $q$-power map on the components of $B_{i}^{n}$. The 
objects of $\operatorname{Vec}(X)$ are viewed as rigid analytic group spaces over $S$, and therefore the object $\mathcal{O}_{X}$ is also called $\mathbb{G}_{a}$.

For an object $E$ of $\operatorname{Vec}(X)$ we let Lie $E$ be the tangent space of the group $E$ along the zero section. Clearly $\operatorname{End}(\operatorname{Lie} E) \cong \operatorname{End}_{\mathcal{O}_{X}}(E)$ and hence there is a diagonal action of $a \in A$ on Lie $E$ by the inclusion $\iota: A \subset K_{\infty} \subset L \subset \Gamma\left(X, \mathcal{O}_{X}\right)$. Furthermore, for every morphism $\alpha: E \rightarrow E^{\prime}$ in $\operatorname{Vec}(X)$ we denote its differential by $\alpha^{\prime}:$ Lie $E \rightarrow$ Lie $E^{\prime}$.

Definition 8.1. Let $X$ be a rigid analytic space over $L$. An abelian A-module $(E, \phi)$ of dimension $d$ and rank $r$ on $X$ consists of a vector bundle $E$ of rank $d$ on $X$ and a ring homomorphism

$$
\phi: A \longrightarrow \operatorname{End}_{\mathbf{V e c}(X)}(E): a \mapsto \phi_{a},
$$

such that the following hold:

(a) There exists an admissible covering $\left\{X_{i}: i \in I\right\}$ of $X$ and natural numbers $n_{i, a}$ for each $a \in A$ satisfying

$$
\left.\left(\iota(a)-\phi_{a}^{\prime}\right)^{n_{i, a}} \operatorname{Lie} E\right|_{X_{i}}=0 .
$$

(b) The sheaf $M(E):=\operatorname{Hom}_{\mathbf{V e c}(X)}\left(E, \mathbb{G}_{a}\right)$, equipped with the action of $a \in A$ by composition on the right with $\phi_{a}$, is a finitely generated $\mathcal{O}_{X} \otimes_{\mathbb{F}_{q}} A$ module (see also Proposition 8.4).

(c) $M(E)$ is a flat $\mathcal{O}_{X}$-module and the fiber of $M(E)$ at every point $x \in X$ is locally free over $k(x) \otimes_{\mathbb{F}_{q}} A$ of rank $r$ (see also Proposition 8.4).

An abelian $A$-module of dimension 1 and rank $r$ is simply a Drinfeld- $A$-module of rank $r$.

We define an operation $\tau$ on $M(E)$ as composition on the left with the Frobenius $\sigma$ on $\mathbb{G}_{a, X}$. This makes $M(E)$ into an algebraic $\tau$-sheaf over $A$ on $X$. Due to the following proposition the rigid analytic $\tau$-sheaves over $A(1)$ and over $A(\infty)$ associated to $M(E)$ are locally free.

As in Anderson [1] one proves:

Proposition 8.2. As $\mathcal{O}_{X} \otimes A$-modules, the dual of Lie $E$, and $M(E) / \tau M(E)$ are isomorphic with $A$ acting on $M(E)$ as defined above and on Lie $E$ via $\phi^{\prime}$.

The following result is clear from the definition:

Proposition 8.3. If $\pi: Y \rightarrow X$ is a general morphism of rigid analytic spaces, then the pullback of an abelian A-module $(E, \phi)$ on $X$ along $\pi$ is an abelian Amodule on $Y$.

We end this section by giving an equivalent condition to (c).

Proposition 8.4. Suppose $\mathcal{M}$ is a finitely generated $\mathcal{O}_{X} \otimes_{\mathbb{F}_{q}} A$-module, where $X=\operatorname{Sp} B$ is an affinoid and $M$ is the $B \otimes_{\mathbb{F}_{q}} A$-module corresponding to $\mathcal{M}$. Then $M$ is locally free over $B \otimes_{\mathbb{F}_{q}} A$ of rank $r$ if and only if $M$ is flat over $B$ and for each $x$ in the set of maximal ideals $\operatorname{Max}(B)$ the module $M \otimes_{B} k(x)$ is locally free over $k(x) \otimes_{\mathbb{F}_{q}}$ A of rank $r$.

Proof. Since one direction is obvious, we now assume that $M$ is flat over $B$ and that $M \otimes_{B} k(x)$ is locally free over $k(x) \otimes A$ for any $x \in \operatorname{Max}(B)$. It suffices to show that for any maximal ideal $\mathfrak{n}$ of $B \otimes A$ the module $M_{\mathfrak{n}}$ is free over $(B \otimes A)_{\mathfrak{n}}$ of rank $r$. 
Since $B$ is Jacobson and $B \otimes A$ is a finitely generated $B$-algebra $\mathfrak{m}:=\mathfrak{n} \cap B$ is a maximal ideal in $B$; cf. [10, Thm. 4.19]. It therefore suffices to show that for each $\mathfrak{m} \in \operatorname{Max}(B)$ the module $M_{\mathfrak{m}}$ is locally free over $B_{\mathfrak{m}} \otimes A$ of rank $r$. Finally because $M$ is finitely generated over $B \otimes A$ and $M \otimes_{B} k(x)$ is locally free over $k(x) \otimes A$ of rank $r$, the proposition is proved once we show that $\operatorname{Tor}_{i}^{B} \otimes A\left(M_{\mathfrak{m}}, N\right)=0$ for all finitely generated $B_{\mathfrak{m}} \otimes A$-modules $N$ and all $i>0$.

By the local criterion of flatness, cf. [22], it suffices to prove the latter assertion for $k(x) \otimes A$-modules $N$ only. For such there is the following spectral sequence:

$$
\operatorname{Tor}_{i}^{k(x) \otimes A}\left(\operatorname{Tor}_{j}^{B_{\mathfrak{m}}}\left(M_{\mathfrak{m}}, k(x)\right), N\right) \Longrightarrow \operatorname{Tor}_{i+j}^{B_{\mathfrak{m}} \otimes A}\left(M_{\mathfrak{m}}, N\right) .
$$

By flatness of $M$ over $B$, the spectral sequence degenerates, and because $M \otimes_{B} k(x)$ is flat over $k(x) \otimes A$, except for $i=j=0$ all terms vanish.

\subsection{Torsion points of abelian $A$-modules.}

Definition 8.5. The étale sheaf $E[\mathfrak{a}]$ on $X$ of $\mathfrak{a}$-torsion points of $(E, \phi)$ is the étale sheaf of $A / \mathfrak{a}$-modules defined for any étale morphism $Y \rightarrow X$ by

$$
E[\mathfrak{a}](Y):=\left\{s \in E(Y): \phi_{a}(s)=0 \text { for every } a \in \mathfrak{a}\right\} .
$$

Theorem 8.6. Let $(E, \phi)$ be an abelian $A$-module on $X$, and suppose $\mathcal{F}$ is the rigid analytic $\tau$-sheaf associated to $M(E)$ as in the first construction of Subsection 1.2. Then there is a natural isomorphism of sheaves of $A / \mathfrak{a}$-modules on $X$

$$
E[\mathfrak{a}] \stackrel{\sim}{\longrightarrow} \underline{\mathcal{F}}[\mathfrak{a}], \quad s \longmapsto\left(h_{s}: f \mapsto f(s)\right) .
$$

Moreover for any ideal $\mathfrak{a}$, the homomorphism $\tau$ on $\underline{\mathcal{F}} / \mathfrak{a} \mathcal{F}$ is an isomorphism, and hence there exists $\mathrm{GL}_{r}(A / \mathfrak{a})$-torsor $\pi: Y \rightarrow X$ so that $\pi^{*} E[\mathfrak{a}]$ is isomorphic to $A / \mathfrak{a}^{r}$.

Proof. For every étale $\pi: Y \rightarrow X$ the morphism

$$
E[\mathfrak{a}](Y) \longrightarrow \underline{\mathcal{F}}[\mathfrak{a}](Y), \quad s \longmapsto\left(h_{s}: f \mapsto f(s)\right) \in \operatorname{Hom}_{\mathcal{O}_{Y}}\left(\pi^{*}(\mathcal{F} / \mathfrak{a} \mathcal{F}), \mathcal{O}_{Y}\right)
$$

is an isomorphism. This can be seen locally on $X$ where $E$ is free by choosing an isomorphism $\kappa: E \stackrel{\sim}{\longrightarrow} \mathcal{O}_{X}^{d}$ and applying $h_{s}$ to the components $\kappa_{i}$ of $\kappa$ which are elements of $\mathcal{F}$.

In view of Theorem 2.5, it remains to prove that $\tau$ is bijective on $\underline{\mathcal{F}} / \mathfrak{a} \underline{\mathcal{F}}$, and it suffices to verify this for principal $\mathfrak{a}=(a)$. For this observe that by condition (a) on an abelian $A$-module the homomorphism $\phi_{a}^{\prime}$ is bijective on Lie $E$. By Proposition 8.2. multiplication by $a$ is bijective on $\mathcal{F} / \tau \mathcal{F}$, i.e., $\mathcal{F}=a \mathcal{F}+\tau \mathcal{F}$. But then $\tau$ is surjective on $\mathcal{F} / a \mathcal{F}$. Since $\mathcal{F} / a \mathcal{F}$ is locally free the asserted bijectivity follows.

8.3. The exponential map. In this section we construct an exponential map for $E$; cf. Anderson [1, $\S 2.1]$. As opposed to working over a field, a difficulty in the relative situation lies in the fact that we may not assume that the $\phi_{a}$ are in Jordan normal form. Therefore we need slightly stronger estimates than Anderson.

Let $E$ be an abelian $A$-module of dimension $d$ on $X$. We choose an affinoid refinement of the covering introduced in (a) of Definition 8.1, such that on each of its open sets $\operatorname{Sp} B$ the sheaf $\left.E\right|_{\operatorname{Sp} B}$ is free. Let $\kappa: \Gamma(\operatorname{Sp} B, E) \rightarrow B^{d}$ be a fixed isomorphism and $\kappa^{\prime}$ the induced isomorphism $\Gamma(\operatorname{Sp} B$, Lie $E) \rightarrow B^{d}$. We call $\kappa$ 
and $\kappa^{\prime}$ coordinates of $\left.E\right|_{\operatorname{Sp} B}$ and Lie $\left.E\right|_{\operatorname{Sp} B}$, respectively. With respect to these coordinates we can represent $\phi_{a}$ and $\phi_{a}^{\prime}$ by matrices

$$
\phi_{a}=\sum_{k=0}^{s_{a}} G_{k}(a) \sigma^{k}, \quad \phi_{a}^{\prime}=G_{0}(a), \quad G_{k}(a) \in M_{d}(B),
$$

where $G_{0}(a)-\iota(a)$ Id is nilpotent and $\sigma$ acts component-wise as the $q$-power map on $B^{d}$. We use the abbreviation $z^{(k)}:={ }^{\sigma^{k}} z$ for vectors, matrices $z$, etc.

Proposition 8.7. There exists a unique sequence of matrices $\left(e_{j}\right)$ in $M_{d}(B)$ with $e_{0}=\operatorname{Id}$ and $\lim _{j \rightarrow \infty} q^{-j} \log \left|e_{j}\right|=-\infty$ defining a rigid analytic function

$$
\exp _{E, \mathrm{Sp} B}: B^{d} \longrightarrow B^{d}, \quad z \mapsto \sum_{j=0}^{\infty} e_{j} z^{(j)},
$$

that satisfies for each $a \in A$ the identity

$$
\exp _{E, \operatorname{Sp} B} \circ \phi_{a}^{\prime}=\phi_{a} \circ \exp _{E, \operatorname{Sp} B} .
$$

The proof is given in Section 8.6 As the functions $\exp _{E, S p} B$ are unique, they agree on overlaps, and so patching yields

Corollary 8.8. Every abelian A-module $E$ on $X$ possesses a unique morphism $\exp _{E}$ : Lie $E \rightarrow E$ of rigid analytic vector bundles, such that for all $a \in A$ one has $\exp _{E} \circ \phi_{a}^{\prime}=\phi_{a} \circ \exp _{E}$, and such that on every affinoid subdomain $\mathrm{Sp} B$ of $X$ which trivializes $E$, and for all coordinates of $\left.E\right|_{\mathrm{Sp} B}$, the morphism $\left.\exp _{E}\right|_{\operatorname{Sp} B}$ takes the form given in Proposition 8.7.

Definition 8.9. For any étale morphism $Y \rightarrow X$ we define

$$
\Lambda(Y):=\Lambda_{E}(Y):=\operatorname{ker}\left(\exp _{E}: \operatorname{Lie} E(Y) \longrightarrow E(Y)\right)
$$

as the kernel of the exponential map on $Y$. This defines an étale sheaf $\Lambda$ of $A_{X^{-}}$ modules on $X$.

For an analytic point $x \in \mathcal{M}(X)$ we consider the pullback $x^{*}(E, \phi)$, which is an abelian $A$-module over $\operatorname{Sp} k(x)$. Its exponential is obtained from $\exp _{E}$ by base change. The kernel of this exponential will be denoted by $\Lambda_{x^{*} E}$.

8.4. The Key-Lemma for abelian $A$-modules. In this section we prove the abelian $A$-module analogue of our Key-Lemma 3.1. It will allow us to show that $\Lambda$ is an overconvergent sheaf and to give in the next section a criterion for uniformizability of abelian $A$-modules.

Key-Lemma 8.10. Let $X=\operatorname{Sp} B$ be affinoid and let $(E, \phi)$ be an abelian $A$ module such that $E \cong \mathcal{O}_{X}^{d}$ is trivial. Let $s \in E(X)$. Let further $x \in \mathcal{M}(X)$ be an analytic point and let $\bar{\ell}$ be a given element of $\left(x^{*} \operatorname{Lie} E\right)\left(k(x)^{\text {alg }}\right)$, defined over an algebraic closure of $k(x)$, satisfying $\exp _{E}(\bar{\ell})=s(x)$.

Then there exists an étale morphism $\pi: V \rightarrow X$ of affinoids such that $\pi V$ is a wide neighborhood of $x$ in $X$, a point $y \in \mathcal{M}(V)$ above $x$, and a uniquely determined section $\ell \in \operatorname{Lie} E(V)$ with $\exp _{E}(\ell)=\left.s\right|_{V}$ and $\ell(y)=\bar{\ell}$. Moreover, if $\bar{\ell}$ is defined over $k(x)$, we can find $V \subset X$ as a wide affinoid neighborhood of $x$. 
Proof. Let $\kappa: E \rightarrow \mathcal{O}_{X}^{d}$ be coordinates on $E$ and let $\rho, \widetilde{\rho} \in L^{\text {alg }}$ with $|\rho|>|\widetilde{\rho}|=$ $\left|\kappa^{\prime}(\bar{\ell})\right|$. Consider

$$
\begin{aligned}
\pi: \quad V & :=\operatorname{Sp} B\left\langle\frac{z_{1}}{\rho}, \ldots, \frac{z_{d}}{\rho}\right\rangle /\left(\exp _{E}\left(z_{1}, \ldots, z_{d}\right)-\kappa(s)\right) \longrightarrow X \quad \text { and } \\
U & :=\left\{y \in V:\left|z_{i}(y)\right| \leq|\widetilde{\rho}|, i=1, \ldots, d\right\} \subset V .
\end{aligned}
$$

Then $U$ is relatively compact in $V$ over $X$, i.e., $U \subset \subset_{X} V$. Furthermore, $\pi$ is étale, since $\exp _{E}(z)=\sum e_{j} z^{(j)}$ with $e_{0}=\mathrm{Id}$; cf. Proposition 8.7. By the choice of $\widetilde{\rho}$ the point $x$ lifts to an analytic point $y$ of $U$ with

$$
\kappa^{\prime}(\bar{\ell})=\left(z_{1}(y), \ldots, z_{d}(y)\right)^{T} .
$$

We set $\ell:=\kappa^{\prime-1}\left(z_{1}, \ldots, z_{d}\right)^{T} \in \operatorname{Lie} E(V)$ and have $\exp _{E}(\ell)=\left.s\right|_{V}$ by construction. By [18, Lemma 3.4.2] $\pi V$ is a wide neighborhood of $x$ in $X$.

If $\bar{\ell}$ is defined over $k(x)$, we have $k(y)=k(x)$. By [18, Lemma 3.1.5] there is an affinoid subdomain $U^{\prime} \subset U$ with $y \in \mathcal{M}\left(U^{\prime}\right)$ such that $\left.\pi\right|_{U^{\prime}}$ is an isomorphism $U^{\prime} \rightarrow \pi\left(U^{\prime}\right)$. Now by [18, Lemma 3.4.2] there is a wide neighborhood $V^{\prime}$ of $y$ such that $\left.\pi\right|_{V^{\prime}}: V^{\prime} \rightarrow \pi V^{\prime}$ is an isomorphism and $\pi V^{\prime}$ is a wide neighborhood of $x$ in $X$.

The central point of the above proof may be restated in terms of Berkovich's theory of analytic spaces as the following result on standard étale neighborhoods for Berkovich spaces:

Proposition 8.11. Let $X=\operatorname{Sp} B$ be affinoid and let $Y:=\operatorname{Sp} B\left\langle T_{1}, \ldots, T_{n}\right\rangle /$ $\left(f_{1}, \ldots, f_{n}\right)$ be étale over $X$. Then $\mathcal{M}(Y)^{\circ}:=\left\{y \in \mathcal{M}(Y):\left|T_{i}\right|_{y}<1\right.$ for all $\left.i\right\}$ is étale over $\mathcal{M}(X)$ in the sense of Berkovich. In particular, the image of $\mathcal{M}(Y)^{\circ}$ in $\mathcal{M}(X)$ is open and thus if $x$ is the image of an analytic point $y$ of $\mathcal{M}(Y)^{\circ}$, then there is an affinoid $V$ of $Y$ whose image is a wide neighborhood of $x$.

Proof. By Berkovich [4, Prop. 3.1.4] the morphism $\mathcal{M}(Y)^{\circ} \rightarrow \mathcal{M}(X)$ is quasi-finite ([4, Def. 3.1.1]) and hence étale ([4, Def. 3.3.4]). The last statement follows from [4, Prop. 3.2.7].

Remark 8.12. The above result is optimal in that one cannot expect $\mathcal{M}(Y)$ to be Berkovich-étale over $\mathcal{M}(X)$. For example consider $X=\operatorname{Sp} L\langle T\rangle$ and $Y=$ $\operatorname{Sp} L\langle T / \pi\rangle$ for $|\pi|<1$. The morphism $\mathcal{M}(Y) \rightarrow \mathcal{M}(X)$ is not quasi-finite on the boundary of $\mathcal{M}(Y)$.

After this digression to Berkovich spaces, let us return to the theory of abelian $A$-modules. As a first application of the above we obtain the following analogue to Corollary 3.2 ,

Corollary 8.13. Let $(E, \phi)$ be an abelian A-module on a connected $X$. If $\pi: Y \rightarrow$ $X$ is a general morphism (cf. Definition A.9), then the map

$$
\Lambda_{E}(X) \rightarrow \Lambda_{\pi^{*} E}(Y), \quad \lambda \mapsto \pi^{*} \lambda
$$

is injective, and its image is a saturated $A$-submodule in $\Lambda_{\pi^{*} E}(Y)$.

Proof. If $X$ and $Y$ are spectra of algebraically closed complete fields $K$ and $L$, the assertion is clear since then we have $\Lambda_{E}(K) \cong \Lambda_{\pi^{*} E}(L)$. As in the proof of Corollary [3.2, it therefore suffices to show the assertion for arbitrary $X$ and for $Y=\operatorname{Sp} \widehat{k(x)^{a l} g}$ for any $x \in \mathcal{M}(X)$, which from now on we assume. 
Since $\lambda \in \Lambda_{E}(X)$ satisfies the étale equation $\exp _{E}(\lambda)=0$ and $X$ is connected, the morphism

$$
h: \Lambda_{E}(X) \longrightarrow \Lambda_{x^{*} E}\left(\widehat{k(x)^{a l} g}\right), \quad \lambda \mapsto \lambda(x)
$$

is injective. To prove that the image is saturated, let $\lambda \in \Lambda_{E}(X), \bar{\mu} \in \Lambda_{x^{*} E}\left(k(x)^{a l g}\right)$ and $a \in A, a \neq 0$, satisfying $\lambda(x)=\phi_{a}^{\prime}(\bar{\mu})$. Since $\phi_{a}^{\prime}$ is an automorphism of Lie $E$, $\bar{\mu}$ is defined over $k(x)$. We let $\mu:=\phi_{a}^{\prime-1}(\lambda) \in \operatorname{Lie} E(X)$ and claim that $\mu \in \Lambda_{E}(X)$.

Choosing an admissible affinoid covering $\operatorname{Sp} B_{i}$ of $X$ we can trivialize $E$. There is an $i$ such that $x$ lies in $\operatorname{Sp} B_{i}$. By Lemma $8.10(s=0)$ there is a wide affinoid neighborhood $V \subset \operatorname{Sp} B_{i}$ of $x$ and a section $\widetilde{\mu} \in \Lambda_{E}(V)$ with $\widetilde{\mu}(x)=\bar{\mu}$. By the injectivity of $h$ we have $\left.\lambda\right|_{V}=\phi_{a}^{\prime}(\widetilde{\mu})$, i.e., $\left.\mu\right|_{V}=\widetilde{\mu}$. Therefore $\left.\exp _{E}(\mu)\right|_{V}=0$. Since $E$ is coherent and $X$ is connected, $E(X) \rightarrow E(V)$ is injective, and we conclude that $\exp _{E}(\mu)=0$ on $X$ as required.

Corollary 8.14. Let $(E, \phi)$ be an abelian A-module on a connected $X$. Then

(a) For every analytic point $x \in \mathcal{M}(X)$ the étale sheaves $x^{*} \Lambda_{E}$ and $\Lambda_{x^{*} E}$ on $\operatorname{Sp} k(x)$ are canonically isomorphic.

(b) For $y \in \mathcal{M}_{\text {ét }}(X)$ the stalk of $\Lambda_{E}$ at $y$ is given by $\Lambda_{y^{*} E}(k(y))$.

(c) $\Lambda_{E}$ is overconvergent on the small étale site of $X$.

(d) The homomorphism $\operatorname{Lie} E \rightarrow E$ is surjective if and only if it is so for the fibers $y^{*} E$ at all étale analytic points $y \in \mathcal{M}_{\text {ét }}(X)$.

The proof is analogous to that of Corollary 3.3. where instead of Lemma 3.1 and Corollary 3.2, one uses Lemma 8.10 and Corollary 8.13, and so it is omitted.

8.5. Uniformizability of abelian A-modules. We now generalize Anderson's notion and characterization of uniformizability of an abelian $A$-module on a point [1, Thm. 4] to the case of an abelian $A$-module on a general base $X$.

Theorem 8.15. Let $E$ be an abelian A-module of dimension d and rank $r$ on $X$. Then the following are equivalent:

(a) The rigid analytic $\tau$-sheaf $\mathcal{F}$ over $A(1)$ on $X$ associated to $E$ is analytically trivial,

(b) there exists a connected temperate étale Galois covering $Z$ of $X$ such that $\Lambda(Z)$ is a projective A-module of rank $r$,

(c) there exists a covering for the étale topology $\left\{Y_{i} \rightarrow X\right\}$ such that $\Lambda\left(Y_{i}\right)$ is a projective $\underline{A}_{X}\left(Y_{i}\right)$-module of rank $r$ for all $i$,

(d) for every analytic point $x \in \mathcal{M}(X)$ the abelian $A$-module $x^{*} E$ is uniformizable in the sense of Anderson,

(e) the following short sequence of étale sheaves on $X$ is exact:

$$
0 \longrightarrow \Lambda \longrightarrow \operatorname{Lie} E \stackrel{\exp _{E}}{\longrightarrow} E \longrightarrow 0 \text {. }
$$

If the equivalent conditions of Theorem 8.15 are satisfied we call $E$ uniformizable.

Proof. $(a) \Rightarrow(b)$. By Theorem 5.10 there exists a connected temperate étale Galois covering $\pi: Z \rightarrow X$, such that $\underline{\mathcal{F}}^{\tau}(Z)$ is a projective $A$-module of rank $r$. As in Anderson one shows that the action $A \rightarrow \operatorname{End}($ Lie $E$ ) extends uniquely to a continuous action of $K_{\infty}$ and that the pairing of $A$-modules

$$
\Lambda(Z) \times \underline{\mathcal{F}}^{\tau}(Z) \longrightarrow \Omega_{A}, \quad(\lambda, f) \mapsto \omega_{\lambda, f},
$$


which is defined by requiring that $f\left(\exp _{E}(z \cdot \lambda)\right)=\operatorname{Res}_{\infty}\left(z \omega_{\lambda, f}\right)$ for all $z \in K_{\infty}$, is perfect. For more details see [6, $\S 9.2]$. We conclude that $\Lambda(Z)$ is a projective $A$-module of rank $r$.

The implications $(b) \Rightarrow(c) \Rightarrow(d) \Rightarrow(a)$ are clear by Corollary 8.13 , Anderson [1, Thm. 4], and Theorem [5.10.

$(d) \Rightarrow(e)$. We have to show that $\exp _{E}$ is surjective, i.e., that for every étale morphism of rigid analytic spaces $Y \rightarrow X$ and every $s \in E(Y)$ there exists a covering for the étale topology $\left\{V_{i} \rightarrow Y\right\}_{i \in I}$ and for each $i$ an element $\ell_{i} \in \operatorname{Lie} E\left(V_{i}\right)$ satisfying $\exp _{E}\left(\ell_{i}\right)=\left.s\right|_{V_{i}}$. By passing to an admissible covering we may assume that $Y=\operatorname{Sp} B$ is a connected affinoid and $\left.E\right|_{Y}$ is trivial. By assumption (d) and Lemma 8.10 every analytic point $y \in \mathcal{M}(Y)$ admits a wide affinoid neighborhood $U_{y}$ and an étale surjective morphism $V_{y} \rightarrow U_{y}$, such that there exists an $\ell_{y} \in \operatorname{Lie} E\left(V_{y}\right)$ with $\exp _{E}\left(\ell_{y}\right)=\left.s\right|_{V_{y}}$. Since $\mathcal{M}(Y)$ is compact, finitely many of the $U_{y}$ suffice, giving the desired covering.

$(e) \Rightarrow(d)$. Let $x \in \mathcal{M}(X)$ be an analytic point. First let $\bar{s} \in x^{*} E\left(k(x)^{s e p}\right)$ be defined over a separable closure $k(x)^{s e p}$ of $k(x)$. As $E$ is coherent the section $\bar{s}$ is actually defined over a finite separable extension $L^{\prime}$ of $k(x)$. By [18, Remark 2.1.2] there is an étale morphism $Y \rightarrow X$ of affinoids and an analytic point $y$ of $Y$ above $x$ with $k(y)=L^{\prime}$. We extend $\bar{s}$ to a section $s \in E(Y)$. Then our assumption (e) implies that there is an étale morphism $V \rightarrow Y$ whose image is a special subset $W$ with $y \in \mathcal{M}(W)$ and an $\ell \in \operatorname{Lie} E(V)$ with $\exp _{E}(\ell)=\left.s\right|_{V}$. Therefore $\bar{\ell}:=\ell(x)$ satisfies $\exp _{E}(\bar{\ell})=\bar{s}$.

Since $k(x)^{s e p}$ is dense in $k(x)^{a l g}$ by $[8,3.4 .1 / 6]$ and $\exp _{E}$ is a local isomorphism we conclude that $\exp _{E}:\left(x^{*} \operatorname{Lie} E\right)\left(k(x)^{\text {alg }}\right) \rightarrow\left(x^{*} E\right)\left(k(x)^{\text {alg }}\right)$ is surjective as required.

\subsection{The proof of Proposition 8.7.}

Proof. Denote by $B\{\{\sigma\}\}$ the ring of formal series $\sum_{k=0}^{\infty} b_{k} \sigma^{k}, b_{k} \in B$, under componentwise addition and composition as multiplication. With this definition, we have $\phi_{a} \in M_{d}(B\{\{\sigma\}\})$. In a first step we construct a formal function $\exp _{E, \mathrm{Sp} B} \in$ $M_{d}(B\{\{\sigma\}\})$ satisfying (8.2). For the argument, we follow Goss [15, p. 75f] which ultimately goes back to Drinfeld. Basically the argument goes by first constructing $\exp _{E, \mathrm{Sp} B}$ which satisfies (8.2) for a single $a \in A \backslash \mathbb{F}_{q}^{a l g}$ and then one shows that the same formal function works for any $a \in A$.

Let $a \in A$ be a non-constant element. Because $G_{0}(a)-a$ is nilpotent, and $a \in A \subset K_{\infty} \subset L$ is a unit in $L$, we have $G_{0}(a) \in \mathrm{GL}_{d}(B)$. A recursive argument, based on the formula $(1+x)^{-1}=\sum x^{n}$, shows that in fact $\phi_{a}$ is invertible in $M_{d}(B\{\{\sigma\}\})$. If follows that $\phi$ may be regarded as a homomorphism

$$
\phi: A \rightarrow M_{d}(B\{\{\sigma\}\}),
$$

with $\phi(A \backslash\{0\}) \subset \mathrm{GL}_{d}(B\{\{\sigma\}\})$. Let us formulate and prove the following lemma:

Lemma 8.16. Let $f=\sum a_{k} \sigma^{k} \in M_{d}(B\{\{\sigma\}\})$. Assume that $a_{0} \in M_{d}(B)$ can be written as $\alpha \mathrm{Id}+N_{1}$ for some $\alpha \in L \backslash \mathbb{F}_{q}^{\text {alg }}$ and some nilpotent matrix $N_{1}$, and suppose that we have $e_{0} \in M_{d}(B)$ with $N_{1} e_{0}=e_{0} N_{2}$ for some nilpotent matrix $N_{2}$. Then there exists a unique $\lambda_{f}=\sum e_{j} \sigma^{j} \in M_{d}(B\{\{\sigma\}\})$ such that

$$
\lambda_{f}\left(\alpha \operatorname{Id}+N_{2}\right)=f \lambda_{f} .
$$


Proof. The conditions on the coefficients $e_{j}$ of $\lambda_{f}$ in formula (8.3) can, by sorting the expression according to powers of $\sigma$, be expressed equivalently in the following recursive way:

$$
\forall j \in \mathbb{N}: e_{j}\left(\alpha^{(j)}+N_{2}^{(j)}\right)=\left(\alpha+N_{1}\right) e_{j}+\sum_{k=1}^{j} a_{k} e_{j-k}^{(k)} .
$$

Because $N_{1}$ and $N_{2}$ are nilpotent and $\alpha^{(j)}-\alpha$ is a unit $\left(\alpha \notin \mathbb{F}_{q}^{a l g}\right)$ for $j>0$, the linear operator

$$
T_{j}: e \mapsto e\left(\alpha^{(j)}+N_{2}^{(j)}\right)-\left(\alpha+N_{1}\right) e
$$

on matrices $e \in M_{d}(B)$ is invertible. By recursively solving for the $e_{j}$ the lemma follows.

We now apply the lemma to $f=\phi_{a}$ and $N_{1}=N_{2}$, and obtain a unique $\lambda_{a} \in$ $M_{d}(B\{\{\sigma\}\})$ whose constant term is the identity matrix Id and such that $\lambda_{a} G_{0}(a)=$ $\phi_{a} \lambda_{a}$. Since the constant term is invertible, $\lambda_{a}$ is a unit in $M_{d}(B\{\{\sigma\}\})$, and so we find

$$
\phi_{a}=\lambda_{a} G_{0}(a) \lambda_{a}^{-1}
$$

As $\phi(A) \subset M_{d}(B\{\{\sigma\}\})$ is commutative, the following is an important observation:

Lemma 8.17. Let $f$ be as in the previous lemma, and assume that $N:=N_{1}=N_{2}$ and $e_{0} \in \mathrm{GL}_{d}(B)$. Then for the centralizer of $f$ in $M_{d}(B\{\{\sigma\}\})$ we have

$$
\operatorname{Cent}_{M_{d}(B\{\{\sigma\}\})}(f)=\lambda_{f} \operatorname{Cent}_{M_{d}(B)}(\alpha \operatorname{Id}+N) \lambda_{f}^{-1} .
$$

Proof. As $f=\lambda_{f}(\alpha \operatorname{Id}+N) \lambda_{f}^{-1}$, any element on the right hand side clearly commutes with $f$. Therefore we only have to show the inclusion $\subseteq$. Suppose that we are given an element $g \in \operatorname{Cent}_{M_{d}(B\{\{\sigma\}\})}(f)$. It is then clear that $\tilde{g}:=\lambda_{f}^{-1} g \lambda_{f}$ belongs to $\operatorname{Cent}_{M_{d}(B\{\{\sigma\}\})}(\alpha \operatorname{Id}+N)$. If we write $\tilde{g}=\sum d_{j} \sigma^{j}$, then this is equivalent to

$$
\forall j>0: T_{j} d_{j}=d_{j}\left(\alpha^{(j)}+N^{(j)}\right)-(\alpha+N) d_{j}=0
$$

and $d_{0}(\alpha \operatorname{Id}+N)=(\alpha \operatorname{Id}+N) d_{0}$. Since the operators $T_{j}$ from the proof of the previous lemma are linear and invertible, we conclude $d_{j}=0$ for $j>0$ and hence $d_{0} \in \operatorname{Cent}_{M_{d}(B)}(\alpha \operatorname{Id}+N)$, as asserted.

By the lemma and the commutativity of $\phi(A)$, we have

$$
\lambda_{a}^{-1} \phi_{a^{\prime}} \lambda_{a} \in \operatorname{Cent}_{M_{d}(B)}\left(G_{0}(a)\right)
$$

for any $a^{\prime} \in A$. Because $\lambda_{a}$ has constant term Id and $\phi_{a^{\prime}}$ has constant term $G_{0}\left(a^{\prime}\right)$ we have in fact

$$
\lambda_{a}^{-1} \phi_{a^{\prime}} \lambda_{a}=G_{0}\left(a^{\prime}\right)
$$

for all $a^{\prime} \in A$. Thus if we fix an element $t \in A \backslash \mathbb{F}_{q}^{\text {alg }}$ and define

$$
\exp _{E, \mathrm{Sp} B}:=\lambda_{t}=\sum_{j} e_{j} \sigma^{j}
$$

then $\exp _{E, \mathrm{Sp} B}$ is the unique element in $M_{d}(B\{\{\sigma\}\})$ with constant term Id which satisfies the condition

$$
\forall a \in A: \quad \exp _{E, \operatorname{Sp} B} G_{0}(a)=\phi_{a} \exp _{E, \operatorname{Sp} B} \in M_{d}(B\{\{\sigma\}\}) .
$$

The proof of the analyticity of $\exp _{E, \mathrm{Sp} B}$ proceeds as in Anderson [1, § 2.1], except that we cannot assume that $G_{0}(t)$ is in Jordan canonical form. So we need 
finer estimates to ensure the convergence of $\exp _{E, \mathrm{Sp} B}$. We fix a complete $L$-algebra norm | $\mid$ on the affinoid algebra $B$. For any matrix $\Delta$ with entries in $B$ we let $|\Delta|$ be the maximum over the norms of the entries. We denote by $\theta$ the image of $t$ in $B$. Observe that $|\theta|>1$. The matrix $N:=G_{0}(t)-\theta$ Id is nilpotent, say with $N^{m+1}=0$. But we don't have $|N| \leq 1$. We set $G_{k}=G_{k}(t)$. The identity (8.4) for $a=t$ implies that the $e_{j}$ are uniquely determined by the recursion

$$
e_{j}=\sum_{k=1}^{s} \sum_{i=0}^{2 m} \sum_{\nu=0}^{i} \frac{\left(\begin{array}{l}
i \\
\nu
\end{array}\right)}{\left(\theta^{q^{j}}-\theta\right)^{i+1}} N^{\nu} G_{k} e_{j-k}^{(k)}\left(N^{(j)}\right)^{i-\nu} .
$$

Thus by induction we see that

$$
e_{j}=\sum_{\underline{k}} \sum_{\underline{i}} \sum_{\underline{\nu}} \frac{(\underline{\underline{i}})}{\theta^{q^{j}(|i|+l)}(1+\epsilon)} H_{j}^{(0)} \cdot \ldots \cdot H_{1}^{(j-1)} e_{0}^{(j)}\left(N^{(j)}\right)^{|i|-|\nu|},
$$

where the first, second and third sum runs over

$$
\begin{aligned}
& \left\{\underline{k}=\left(k_{1}, \ldots, k_{j}\right) \in \mathbb{N}_{0}^{j}: k_{\mu} \leq s, k_{j} \neq 0, \text { and } k_{1}+\ldots+k_{\mu}=\mu \text { if } k_{\mu} \neq 0\right\}, \\
& \left\{\underline{i}=\left(i_{1}, \ldots, i_{j}\right) \in \mathbb{N}_{0}^{j}: i_{\mu} \leq 2 m, \text { and } i_{\mu}=0 \text { if } k_{\mu}=0\right\}, \\
& \left\{\underline{\nu}=\left(\nu_{1}, \ldots, \nu_{j}\right) \in \mathbb{N}_{0}^{j}: \nu_{\mu} \leq i_{\nu}\right\},
\end{aligned}
$$

respectively, where we abbreviate

$$
\begin{aligned}
& \left(\begin{array}{l}
\underline{i} \\
\underline{\nu}
\end{array}\right)=\left(\begin{array}{c}
i_{1} \\
\nu_{1}
\end{array}\right) \cdot \ldots \cdot\left(\begin{array}{c}
i_{j} \\
\nu_{j}
\end{array}\right) \\
& |i|=i_{1}+\ldots+i_{j}, \quad|\nu|=\nu_{1}+\ldots+\nu_{j}, \\
& l=l(\underline{k})=\#\left\{\mu: k_{\mu} \neq 0\right\} \text {, } \\
& H_{\mu}=H_{\mu}(\underline{k}, \underline{\nu})= \begin{cases}\mathrm{Id} & \text { if } \quad k_{\mu}=0, \\
N^{\nu_{\mu}} G_{k_{\mu}} & \text { if } \quad k_{\mu} \neq 0,\end{cases}
\end{aligned}
$$

and where $\epsilon=\epsilon(\underline{k}, \underline{i}, \underline{\nu})$ is a polynomial in $\theta^{-1}$ with $|\epsilon|<1$. Now the constraints on $\underline{k}$ imply that $l \geq j / s$. Hence the absolute value of the fraction is at most

$$
|\theta|^{-q^{j} l} \leq|\theta|^{-q^{j} j / s} .
$$

Furthermore, if $|i|-|\nu|>m$, the most right factor is zero. Let $M$ be a common upper bound for $\left|G_{\mu}\right|,|N|, \ldots,\left|N^{m}\right|$. Thus we see that

$$
\left|H_{\mu}^{(j-\mu)}\right| \leq M^{2 q^{j-\mu}} \quad \text { and } \quad\left|\left(N^{(j)}\right)^{|i|-|\nu|}\right| \leq M^{q^{j}} .
$$

Thus we conclude as required

$$
q^{-j} \log \left|e_{j}\right| \leq-j / s \log |\theta|+q^{-j} \frac{q^{j}-1}{q-1} \log M^{2}+\log M \quad \longrightarrow-\infty \quad(j \rightarrow \infty) .
$$

This proves Proposition 8.7 


\section{Appendix A. Review of analytic Spaces And Étale Sheaves}

A.1. Analytic points. Given a category of sheaves of abelian groups on a space $X$ one wants to dispose of a sufficiently large set of points to detect exactness via stalks at these points. On a rigid analytic space $X$ the classical points (the ones usually considered in rigid analytic geometry, whose residue fields are finite extensions of the base field) are too few if one works with sheaves which are not coherent. To remedy this, van der Put 23 introduced the larger class of analytic points together with overconvergent sheaves (Definitions A.1 and A.12 below). In the category of overconvergent sheaves on $X$ a sequence is exact if and only if for every analytic point $x$ of $X$ the associated sequence of stalks at $x$ is exact; cf. [25, $\S 2]$ or [18, $\S 2]$.

An important tool in the study of uniformizability of $\tau$-sheaves are their associated sheaves of $\tau$-invariants. The latter will turn out to be overconvergent (étale) sheaves; cf. Corollary 3.3. We therefore now briefly recall the concepts of analytic point, overconvergent (étale) sheaf, etc., and describe some basic properties. For more details see the articles [3], 18, [23, 24, 25] by Berkovich, de Jong, van der Put, and Schneider.

Let $L$ be a complete, non-archimedean valued field and denote by $X=\operatorname{Sp} B$ an affinoid rigid analytic space over $L$. Following [18, we call an admissible subset $V \subset X$ special if $V=\bigcup V_{i}$ is a finite union of affinoid subdomains $V_{i} \subset X$.

Definition A.1. An analytic point $x$ of $X$ is a semi-norm $|\cdot|_{x}: B \rightarrow \mathbb{R}_{\geq 0}$ which satisfies:

(a) $|f+g|_{x} \leq \max \left\{|f|_{x},|g|_{x}\right\} \quad$ for all $f, g \in B$,

(b) $|f g|_{x}=|f|_{x}|g|_{x}$ for all $f, g \in B$,

(c) $|\lambda|_{x}=|\lambda| \quad$ for all $\lambda \in L$,

(d) $\mid$. $\left.\right|_{x}: B \rightarrow \mathbb{R}_{\geq 0}$ is continuous with respect to the norm topology on $B$.

The set of all analytic points of $X$ is denoted $\mathcal{M}(X)$.

On $\mathcal{M}(X)$ one considers the coarsest topology such that for every $f \in B$ the map $\mathcal{M}(X) \rightarrow \mathbb{R}$ given by $x \mapsto|f|_{x}$ is continuous. Equipped with this topology, $\mathcal{M}(X)$ is a compact Hausdorff space; cf. [3, Thm. 1.2.1], [23, § 1] or [25, §1].

Every classical point $x \in X$ defines a semi-norm as above via

$$
B \longrightarrow k(x) \stackrel{|\cdot|_{x}}{\longrightarrow} \mathbb{R}_{\geq 0}
$$

where $|\cdot|_{x}$ is the unique norm on its residue field $k(x)$ which extends the norm of $L$. Thereby one obtains an injective map $X \rightarrow \mathcal{M}(X)$. With respect to the canonical topology on $X$, cf. $[8, \S 7.2 .1$, this map is a homeomorphism onto a dense subset in $\mathcal{M}(X)$; cf. [3, Prop. 2.1.15].

For a classical point $x \in X$ the residue field $k(x)=B / \operatorname{ker}|\cdot|_{x}$ is a finite extension of $L$ and thus complete with respect to $|\cdot|_{x}$. For an arbitrary analytic point $x$ in $\mathcal{M}(X)$ this is no longer true. In this situation one defines $k(x)$ to be the completion of the fraction field of $B / \operatorname{ker}|\cdot|_{x}$ with respect to $|\cdot|_{x}$ and calls it the (complete) residue field of $x$. This gives rise to a continuous homomorphism $B \rightarrow k(x)$ of $L$-algebras. Hence the analytic points coincide with the equivalence classes of analytic points defined by Schneider [25, §1].

Every morphism $f: Y \rightarrow X$ of affinoid rigid analytic spaces over $L$ induces a continuous morphism $\mathcal{M}(f): \mathcal{M}(Y) \rightarrow \mathcal{M}(X)$ by mapping the semi-norm $\mathcal{O}(Y) \rightarrow$ $\mathbb{R}_{\geq 0}$ to the composition $\mathcal{O}(X) \rightarrow \mathcal{O}(Y) \rightarrow \mathbb{R}_{\geq 0}$. In particular, for an affinoid subdomain $U \subset X$ this morphism identifies $\mathcal{M}(U)$ with a closed subset of $\mathcal{M}(X)$. 
Definition A.2. If $U \subset X$ is an admissible subset with an admissible affinoid covering $\left\{U_{i}\right\}$ one defines

$$
\mathcal{M}(U):=\bigcup_{i} \mathcal{M}\left(U_{i}\right) \subset \mathcal{M}(X) .
$$

It follows from [25. Lemma 1.3] that this definition is independent of the chosen covering $\left\{U_{i}\right\}$. In particular, if $U$ is itself affinoid one recovers Definition A.1,

Definition A.3. An admissible subset $U \subset X$ is called a wide neighborhood of an analytic point $x \in \mathcal{M}(X)$ if $x$ lies in the interior $\mathcal{M}(U)^{\circ}$.

Note that if $x \in X$ is a classical point, then every admissible $U \subset X$ containing $x$ is a wide neighborhood of $x$. However, for general analytic points this is not the case.

Definition A.4. For admissible subsets $V \subset U \subset X$ one calls $U$ a wide neighborhood of $V$ in $X$ and writes $V \subset \subset_{X} U$ if $\mathcal{M}(V)$ is contained in the interior $\mathcal{M}(U)^{\circ}$.

If $V$ and $U$ are affinoid, Kiehl [20, Def. 2.1] originally used the symbol $V \subset \subset_{X} U$ to mean that $V$ is relatively compact in $U$ over $X$, i.e., that there is an affinoid generating system $f_{1}, \ldots, f_{r}$ of $\mathcal{O}_{X}(U)$ over $\mathcal{O}_{X}(X)$ such that

$$
V \subset\left\{x \in U:\left|f_{i}(x)\right|<1 \text { for } 1 \leq i \leq r\right\} .
$$

By the following result, Kiehl's definition is equivalent to the one given in Definition $\mathrm{A.4}$.

Proposition A.5. Let $V \subset U \subset X$ be admissible subsets.

a) If $V$ and $U$ are affinoid, then $U$ is a wide neighborhood of $V$ in $X$ if and only if $V$ is relatively compact in $U$ over $X$.

b) If $V=\bigcup V_{i}$ is special, then $V \subset \subset_{X} U$ if and only if $V_{i} \subset \subset_{X} U$ for every $i$.

c) If $V$ is special, then $V \subset \subset_{X} U$ if and only if there exists a special subset $W \subset U$ such that $V \subset \subset_{X} W$.

d) $\mathcal{M}(U)^{\circ}=\bigcup\left\{\mathcal{M}(V): V\right.$ affinoid with $\left.V \subset \subset_{X} U\right\}$.

Proof. Part (a) is from [25, Prop. 1.23], part (b) follows immediately from the definition, and (c) and (d) are from [25, Remark 1.24] and [25, Lemma 1.12], respectively.

The following two propositions are taken from [25, Prop. 3.3 and Lemma 3.1], and [25, Prop. 3.5], respectively.

Proposition A.6. For a special subset $V \subset X$, the set $\mathcal{M}(X \backslash V)=\mathcal{M}(X) \backslash \mathcal{M}(V)$ is open in $\mathcal{M}(X)$.

Proposition A.7. Let $U=\bigcup\left\{U_{i}: i \in I\right\}$ be a covering of an admissible subset $U \subset X$ by admissible subsets $U_{i} \subset X$ with $\mathcal{M}\left(U_{i}\right) \subset \mathcal{M}(X)$ open for all $i$. Then this covering is admissible if and only if $\mathcal{M}(U)=\bigcup\left\{\mathcal{M}\left(U_{i}\right): i \in I\right\}$. In this case $\mathcal{M}(U)$ is open in $\mathcal{M}(X)$.

We further need the following lemma. 
Lemma A.8. Let $X$ be an affinoid rigid analytic space and let $X_{i}, i \in I$, be a finite affinoid covering of $X$. Let $M \subset \mathcal{M}(X)$ be a subset. Then $M$ is open in $\mathcal{M}(X)$ if and only if $M \cap \mathcal{M}\left(X_{i}\right)$ is open in $\mathcal{M}\left(X_{i}\right)$ for every $i$.

Proof. This is a standard argument in point set topology, which we repeat for sake of completeness: Define $M^{c}:=\mathcal{M}(X) \backslash M$. Then we need to show that $M^{c}$ is closed in $\mathcal{M}(X)$ if and only if $M^{c} \cap \mathcal{M}\left(X_{i}\right)$ is closed for every $i$. This however is clear since the compact sets $\mathcal{M}\left(X_{i}\right)$ form a finite cover of the Hausdorff space $\mathcal{M}(X)$ by closed subsets.

The notion of analytic point can be defined for general rigid analytic spaces $X$ over $L$. Namely, if $\left\{X_{i}\right\}_{i \in I}$ is an admissible affinoid covering of $X$, the analytic points of $X$ are just the analytic points of the $X_{i}$ modulo the obvious identification coming from the inclusions $X_{i} \cap X_{j} \subset X_{i}, X_{j}$. See [25, §2] for a precise definition. The definition of a suitable topology on $\mathcal{M}(X)$, so that the resulting space is Hausdorff again, is more subtle.

If $X$ is quasi-separated and admits a locally finite admissible affinoid covering $\left\{X_{i}\right\}$ this can be done as follows: (Recall that $X$ is called quasi-separated if the intersection of any two admissible affinoid subsets of $X$ is a finite union of affinoid subsets and that a covering $\left\{X_{i}\right\}$ is called locally finite if each $X_{i}$ meets only finitely many $X_{j}$.) One defines

$$
\mathcal{M}(X):=\bigcup \mathcal{M}\left(X_{i}\right) / \sim
$$

where $\sim$ is the equivalence relation obtained by gluing $\mathcal{M}\left(X_{i}\right)$ and $\mathcal{M}\left(X_{j}\right)$ along the subset $\mathcal{M}\left(X_{i} \cap X_{j}\right)$. The topology on $\mathcal{M}(X)$ is the finest for which all the natural inclusions $\mathcal{M}\left(X_{i}\right) \hookrightarrow \mathcal{M}(X)$ are continuous. For such $X$ Lemma A.8 is still true; cf. [24, Lemma 5.3].

For the general situation V.G. Berkovich in 3], 4], has developed a theory of analytic spaces using the compact spaces $\mathcal{M}(X)$ for affinoid $X$ as building blocks. However, the category of analytic spaces he obtains is different from the category of all rigid analytic spaces. As we want to remain within the framework of rigid analytic spaces, we therefore speak of $\mathcal{M}(X)$ only for rigid analytic spaces $X$ which are quasi-separated and paracompact. Recall that a rigid analytic space is called paracompact if every admissible covering admits a locally finite refinement.

A.2. General morphisms. We recall the notion of general morphisms introduced by de Jong, van der Put [18, § 2.6]. Consider an extension of complete valued fields $L \subset L^{\prime}$. In [8, $\left.\S 9.3 .6\right]$ there is constructed a base change functor $X \mapsto X \widehat{\otimes}_{L} L^{\prime}$ from quasi-separated rigid analytic $L$-spaces to quasi-separated rigid analytic $L^{\prime}$-spaces.

Definition A.9. Let $L \subset L^{\prime}$ be an extension of complete valued fields and let $X$ and $Y$ be rigid analytic spaces over $L$ and $L^{\prime}$, respectively, with $X$ quasi-separated. A general morphism $f: Y \rightarrow X$ is a morphism $f: Y \rightarrow X \widehat{\otimes}_{L} L^{\prime}$ of rigid analytic spaces over $L^{\prime}$.

If both $X$ and $Y$ are affinoid, then this is simply a continuous homomorphism $\mathcal{O}(X) \rightarrow \mathcal{O}(Y)$, since any such homomorphism factors as

$$
\mathcal{O}(X) \rightarrow \mathcal{O}(X) \widehat{\otimes}_{L} L^{\prime} \rightarrow \mathcal{O}(Y)
$$


If $Y \rightarrow X$ is a general morphism, and $Z \rightarrow X$ is a morphism of rigid analytic spaces over $L$, then we can form the fiber product

$$
Y \times_{X} Z:=Y \times_{X \widehat{\otimes}_{L} L^{\prime}} Z \widehat{\otimes}_{L} L^{\prime}
$$

Every general morphism $f: Y \rightarrow X$ gives rise to a pullback functor $f^{*}$ from (quasi-)coherent $\mathcal{O}_{X}$-modules to (quasi-)coherent $\mathcal{O}_{Y}$-modules. Any analytic point $x \in \mathcal{M}(X)$ can be viewed as a general morphism $i: \operatorname{Sp} k(x) \rightarrow X$.

A.3. Étale sheaves on rigid analytic spaces. A morphism $f: Y \rightarrow X$ of rigid analytic spaces over $L$ is called étale if for every (classical) point $y \in Y$ the induced homomorphism of local rings $\mathcal{O}_{X, f(y)} \rightarrow \mathcal{O}_{Y, y}$ is flat and unramified. See [18, § 3] for a thorough discussion of this notion.

Let $X$ be a rigid analytic space over $L$. We recall the definition of the étale site of $X$ from Schneider, Stuhler [26, p. 58]; cf. also [18, $\S 3.2$ ]. The underlying category of the site $X_{\text {étale }}$ is the category of all étale morphisms $f: Y \rightarrow X$ of rigid analytic spaces over $L$. A morphism from $f$ to $f^{\prime}$ is a morphism $g: Y \rightarrow Y^{\prime}$ such that $f^{\prime} \circ g=f$. The morphism $g$ is automatically étale.

Definition A.10. A family of étale morphisms $\left\{g_{i}: Z_{i} \rightarrow Y\right\}_{i \in I}$ is a covering for the étale topology if it has the following property:

For every (some) choice of admissible affinoid covering $Z_{i}=\bigcup_{j} Z_{i, j}$ one has $Y=\bigcup_{i, j} g_{i}\left(Z_{i, j}\right)$, and this is an admissible covering in the Grothendieck-topology of $Y$.

Clearly any admissible covering of $Y$ is a covering for the étale topology.

The property in Definition A.10 is local on $Y$ in the following sense: if $Y=\bigcup Y_{l}$ is an admissible affinoid covering, then $\left\{g_{i}: Z_{i} \rightarrow Y\right\}$ is a covering for the étale topology if and only if for all $l$ the same is true for the covering $\left\{g_{i}: g_{i}^{-1}\left(Y_{l}\right) \rightarrow Y_{l}\right\}$. This implies that if $\left\{Z_{i} \rightarrow Y\right\}$ and $\left\{W_{i, j} \rightarrow Z_{i}\right\}$ for all $i$ are coverings for the étale topology, then $\left\{W_{i, j} \rightarrow Y\right\}$ is a covering for the étale topology.

The category $X_{\text {étale }}$ equipped with the family of coverings for the étale topology is thus a site, called the étale site of $X$. The sheaves on this site are called étale sheaves on $X$.

If $\left\{Z_{i} \rightarrow Y\right\}$ is a covering for the étale topology, and $Y^{\prime} \rightarrow Y$ is a general morphism, then the fiber product $\left\{Y^{\prime} \times_{Y} Z_{i} \rightarrow Y^{\prime}\right\}$ is a covering for the étale topology; cf. [18, Lemma 3.2.1]. So every general morphism $f: X^{\prime} \rightarrow X$ induces a morphism of sites $X_{\text {étale }}^{\prime} \rightarrow X_{\text {étale }}$.

Examples A.11. The following are examples of étale sheaves on $X$ which we will need in the sequel $\left(f: Y \rightarrow X\right.$ will denote a general object of $\left.X_{\text {étale }}\right)$ :

a) The structure sheaf $\mathbb{G}_{a}$ defined by $Y \mapsto \Gamma\left(Y, \mathcal{O}_{Y}\right)$.

b) For any quasi-coherent sheaf $\mathcal{F}$ of $\mathcal{O}_{X}$-modules on $X$ we define the the étale sheaf $W(\mathcal{F})$ on $X_{\text {étale }}$ by $Y \mapsto \Gamma\left(Y, f^{*} \mathcal{F}\right)$, where $f^{*}$ denotes the pullback of quasi-coherent modules. In particular, one has $W\left(\mathcal{O}_{X}\right) \cong \mathbb{G}_{a}$. Any étale sheaf $W(\mathcal{F})$ is a sheaf of $\mathbb{G}_{a}$-modules.

c) Any representable sheaf $Y \mapsto \operatorname{Hom}_{X}(Y, Z)$ given by some rigid analytic space $Z$ over $X$.

d) For any group or ring $B$ the constant étale sheaf $\underline{B}_{X}$ is defined by $Y \mapsto$ $\prod_{\pi_{0}(Y)} B$, where $\pi_{0}(Y)$ is the set of connected components of $Y$. (The restriction maps are the obvious ones.) The sheaf $\underline{B}_{X}$ is in fact representable, 
namely by $\coprod_{b \in B} X$. If $X$ is clear from the context we will also write $\underline{B}$ instead of $\underline{B}_{X}$.

Definition A.12. A sheaf $\mathcal{S}$ on $X_{\text {étale }}$ is called overconvergent if for every $Y \rightarrow X$ in $X_{\text {étale }}$ with $Y$ affinoid the following holds:

For every special subset $V \subset Y$ we have $\mathcal{S}(V)=$

$$
\begin{aligned}
& \lim _{V \overrightarrow{\subset_{Y}} U} \mathcal{S}(U) \text {, where the limit is taken over all special } \\
& \text { subsets } U \subset Y \text { with } V \subset \subset_{Y} U .
\end{aligned}
$$

The following is taken from [18, $\S 3$ ]: An étale sheaf $\mathcal{S}$ is overconvergent if and only if for every $Y \rightarrow X$ in $X_{\text {étale }}$ there exists an admissible affinoid covering $Y_{i}, i \in$ $I$, of $Y$ such that $(*)$ holds on each $Y_{i}$. The full subcategory of overconvergent étale sheaves in the category of all abelian étale sheaves is closed under the formation of quotients, subsheaves and extensions, i.e., it is a Serre subcategory. The space of étale analytic points of $X$ is defined as

$$
\mathcal{M}_{\text {ét }}(X):=\lim _{\longrightarrow}\{\mathcal{M}(Y): \text { for } Y \rightarrow X \text { étale }\} .
$$

In line with [18, $\S 3.3]$, étale points $y$ can be assigned a complete, algebraically closed residue field $k(y)$. We also regard $y$ as the general morphism $y: \operatorname{Sp} k(y) \rightarrow X$.

For an étale sheaf $\mathcal{S}$ and $y \in \mathcal{M}_{\text {ét }}(X)$ one may define a stalk $\mathcal{S}_{y}$. Étale analytic points are sufficient to detect exactness in short exact sequences of overconvergent étale sheaves.

A.4. Fundamental groups. In this section we want to recall de Jong's [17] definition of the étale fundamental group and André's [2, § III.2] definition of the temperate étale fundamental group of a rigid analytic space. These concepts are best defined using Berkovich's analytic spaces; cf. [3, 4. To stay within the framework of rigid analytic spaces, we therefore assume throughout this section that all rigid analytic spaces are quasi-separated and paracompact.

Let $X$ be an affinoid rigid analytic space over a complete, non-archimedean valued field $L$.

Definition A.13. Let $\pi: Y \rightarrow X$ be a morphism of rigid analytic spaces over $L$.

a) $Y$ is called an étale covering space of $X$ if every analytic point $x$ of $X$ has a wide affinoid neighborhood $U$ such that $Y \times_{X} U$ is a disjoint union of affinoids $V_{i}$ with $\left.\pi\right|_{V_{i}}: V_{i} \rightarrow U$ finite étale.

b) $Y$ is called a topological covering space of $X$ if we can choose $U$ and the $V_{i}$ above such that $\left.\pi\right|_{V_{i}}: V_{i} \rightarrow U$ is an isomorphism.

c) $Y$ is called a finite étale covering space of $X$ if $\pi$ is finite étale.

The category of topological coverings is equivalent to the category of covering spaces of the topological Hausdorff space $\mathcal{M}(X)$; cf. [17, Lemma 2.6]. The finite étale covering spaces are used in algebraic geometry to define the algebraic fundamental group [SGA 1].

Definition A.14. Let $Y$ be an étale covering space of $X$. $Y$ is called a temperate étale covering space of $X$ if there exists a finite étale covering space $S$ of $X$ and a topological covering space $T$ of $S$ such that $Y$ is the quotient of $T$ by an equivalence relation $R \subset T \times_{X} T$ which is a union of connected components. 
All the above notions of covering spaces are stable under taking connected components, fiber products and quotients.

Clearly algebraic and topological coverings are temperate, and temperate coverings are étale. Furthermore, all étale covering spaces are coverings for the étale topology in the site $X_{\text {étale }}$; cf. Definition A.10. The reader should not confuse the concepts of étale covering spaces and coverings for the étale topology.

\section{ACKNOWLEDGEMENTS}

We would both like to thank R. Pink for his interest in this work, his example and many related mathematical discussions. Our thanks also go to the anonymous referee for some insightful comments that led to a simplification in the proof of Proposition 7.1 .

\section{REFERENCES}

[SGA 1] A. Grothendieck: Revêtements étales et groupe fondamentale, LNM 224, SpringerVerlag, Berlin-Heidelberg 1971. MR0354651(50:7129)

[SGA 3] M. Demazure, A. Grothendieck: Schémas en Groupes I, II, III, LNM 151, 152, 153, Springer-Verlag, Berlin-Heidelberg 1970.

[1] G.W. Anderson: t-motives, Duke Math. J. 53 (1986), 457 - 502. MR0850546 (87j:11042)

[2] Y. André: Period mappings and differential equations; from $\mathbb{C}$ to $\mathbb{C}_{p}$, MSJ Memoirs 12, Math. Soc. Japan, Tokyo 2003. MR1978691 (2004g:14014)

[3] V.G. Berkovich: Spectral theory and analytic geometry over non-Archimedean fields, Mathematical Surveys and Monographs 33, Amer. Math. Soc., Providence 1990. MR $1070709(91 \mathrm{k}: 32038)$

[4] V.G. Berkovich: Étale cohomology for non-Archimedean analytic spaces, Publ. Math. I.H.E.S. 78 (1993), 5 - 161. MR.1259429 (95c:14017)

[5] G. Böckle: Global $L$-functions over function fields, Math. Ann. 323 (2002), no. 4, 737795. MR.1924278 (2003e:11052)

[6] G. Böckle: An Eichler-Shimura isomorphism over function fields between Drinfeld modular forms and cohomology classes of crystals, preprint 2001, available under: http://www.exp-math.uni-essen.de/ boeckle .

[7] G. Böckle, R. Pink: A cohomological theory of crystals over function fields, in preparation.

[8] S. Bosch, U. Güntzer, R. Remmert: Non-archimedean analysis, Springer-Verlag, BerlinHeidelberg 1984. MR0746961 (86b:32031)

[9] V.G. Drinfeld: Moduli variety of F-sheaves, Functional Anal. Appl. 21 (1987), no. 2, 107-122. MR0902291 (89b:11092)

[10] D. Eisenbud: Commutative Algebra with a View towards Algebraic Geometry, SpringerVerlag, New York, 1994. MR.1322960 (97a:13001)

[11] S. Bosch, W. Lütkebohmert: Formal and Rigid Geometry I. Rigid Spaces, Math. Ann. 295 (1993), 291 - 317. MR.1202394 (94a:11090)

[12] F. Gardeyn: t-Motives and Galois Representations, Dissertation Universiteit Gent, Oct. 2001.

[13] F. Gardeyn: A Galois criterion for good reduction of $\tau$-sheaves, J. Number Theory 97 (2002), 447 - 471. MR1942970(2003i:11074)

[14] F. Gardeyn: New criteria for uniformization of t -motives. Preprint 2001.

[15] D. Goss: Basic Structures of Function Field Arithmetic, Ergebnisse 35, Springer-Verlag, Berlin-Heidelberg-New York 1996. MR.1423131 (97i:11062)

[16] U. Hartl: Uniformizing the Stacks of Abelian Sheaves, in Number Fields and Function fields - Two Parallel Worlds, Papers from the 4th Conference held on Texel Island, April 2004, eds. G. van der Geer, B. Moonen, R. Schoof, pp. 167 - 222, Progress in Math. 239, Birkhäuser, Basel 2005. See also arXiv:math.NT/0409341. MR2176591 (2006k:11106)

[17] J. de Jong: Étale Fundamental groups of non-Archimedean analytic spaces, Comp. Math. 97 (1995), 89 - 118. MR1355119 (97c:32047) 
[18] J. de Jong, M. van der Put: Étale Cohomology of Rigid Analytic Spaces, Doc. Math. 1 (1996), 1 - 56. MR.1386046 (98d:14024)

[19] N. Katz, B. Mazur: Arithmetic Moduli of Elliptic Curves, Ann. Math. Stud. 108, Princeton University Press, Princeton 1985. MR0772569 (86i:11024)

[20] R. Kiehl: Der Endlichkeitssatz für eigentliche Abbildungen in der nichtarchimedischen Funktionentheorie, Invent. Math. 2 (1967), 191 - 214. MR0210948 (35:1833)

[21] W. Lütkebohmert: Vektorraumbündel über nichtarchimedischen holomorphen Räumen, Math. Z. 152 (1977), 127 - 143. MR0430331(55:3336)

[22] H. Matsumura: Commutative ring theory, Cambridge University Press, Cambridge 1989. MR.1011461 (90i:13001)

[23] M. van der Put: Cohomology on affinoid spaces, Comp. Math. 45 (1982), 165 - 198. MR0651980 (83g:32014)

[24] M. van der Put, P. Schneider: Points and topologies in rigid geometry, Math. Ann. 302 (1995), 81 - 103. MR1329448 (96k:32070)

[25] P. Schneider: Points of rigid analytic varieties, J. reine Angew. Math. 434 (1993), 127 157. MR 1195693 (94b:14017)

[26] P. Schneider, U. Stuhler: The cohomology of $p$-adic symmetric spaces, Invent. Math. 105 (1991), 47 - 122. MR:1109620 (92k:11057)

[27] Y. Taguchi, D. Wan: L-functions of $\phi$-sheaves and Drinfeld modules, J. Amer. Math. Soc. 9 (1996), no. 3, 755 - 781. MR1327162 (96j:11082)

Institut für Experimentelle Mathematik, Universität Duisburg-Essen, Campus Essen, Ellernstr. 29, D-45326 Essen, Germany

E-mail address: boeckle@iem.uni-due.de

Mathematisches Institut, Albert-Ludwigs-Universität Freiburg, Eckerstr. 1, D 79104 Freiburg, Germany

E-mail address: urs.hartl@math.uni-freiburg.de 